FSU-HEP-20000920

\title{
High-order corrections and subleading logarithms for top quark production
}

\author{
Nikolaos Kidonakis \\ Physics Department \\ Florida State University \\ Tallahassee, FL 32306-4350, USA
}

\begin{abstract}
We derive high-order threshold corrections for top quark production in hadronic collisions from resummation calculations. We present analytical expressions for the cross section through next-to-next-to-next-to-next-to-leading order $\left(\mathrm{N}^{4} \mathrm{LO}\right)$ and next-to-nextto-leading logarithmic accuracy. Special attention is paid to the role of subleading logarithms and how they relate to the convergence of the perturbation series and differences between various resummation prescriptions. It is shown that care must be taken to avoid unphysical terms in the expansions. Numerical results are presented for top quark production at the Tevatron. We find sizeable increases to the total cross section and differential distributions and a dramatic reduction of the factorization scale dependence relative to next-to-leading order.
\end{abstract}




\section{Introduction}

The top quark production cross section at the Tevatron receives significant contributions from the threshold region, where there is limited phase space for the emission of real gluons. The incomplete cancellation of infrared divergences between real and virtual graphs produces finite, but large, logarithmic corrections in the form of "plus" distributions. These corrections can be resummed to all orders in the perturbative expansion.

The need for threshold resummations was recognized over a decade ago for the Drell-Yan cross section [1, 2]. The resummation of the leading logarithms for heavy quark production [3, 4. 5] relies heavily on this work since these logarithms are universal between electroweak and QCD induced cross sections. To go beyond leading logarithms one has to take into account the complex color structures of QCD cross sections [6, 7]. Resummation for heavy quark production at next-to-leading logarithmic (NLL) accuracy was first presented in Refs. [6, [7] and then in Refs. [8, 9, 10, 11, 12]. For a review see Refs. [13, 14.

Threshold resummation follows from the factorization properties of the cross section [15], separating long- and short-distance physics. The hadronic cross section is written as a convolution of non-perturbative parton distribution functions with the perturbative partonic cross section. This convolution becomes a simple product if one takes moments of the cross section. The perturbative cross section still has sensitivity to soft-gluon dynamics and is a smooth function only away from the edges of partonic phase space. In general it includes "plus" distributions with respect to a variable that measures distance from partonic threshold. It is these singular distributions that can be resummed to all orders in perturbation theory. This is achieved by first refactorizing the cross section [7] into new functions which absorb the universal collinear singularities associated with the incoming partons, and a function that describes non-collinear soft gluon emission. Resummation is explicitly derived in moment space from the renormalization group properties of these functions [7, 11, 16, 17]. To obtain the physical resummed cross section the moment space results must be inverted back to momentum space. There have been a few proposals or "prescriptions" for the best way to do the inversion or otherwise use the resummation [3, 4, 5]. Numerically, the choice of prescription can have a significant effect. Alternatively, the resummed cross section can be used as a generator of perturbation theory. At fixed order no prescription is necessary to invert the moment-space results. This is the approach taken in this paper.

The aim of this paper is two-fold. First, to derive the expansion of the resummed cross section through next-to-next-to-next-to-next-to-leading order $\left(\mathrm{N}^{4} \mathrm{LO}\right)$ and next-to-next-to-leading logarithmic (NNLL) accuracy; and second, to investigate and assess the importance of subleading logarithms (beyond NNLL). We calculate the numerical effect of these higher orders and subleading logarithms for top quark production at the Tevatron. As we will see, this discussion intimately relates to the convergence of the perturbation series as well as to the choice of a resummation prescription, over which there have been different viewpoints [5, 18]. We will show that one has to be careful to avoid unphysical terms in the fixed-order expansions. These considerations apply to QCD hard scattering cross sections in general. We find large corrections for top quark production at the Tevatron and a dramatically reduced factorization scale dependence. Our formalism allows us to make predictions for both total and differential cross sections.

In a companion paper 19 the next-to-leading order (NLO) and next-to-next-to-leading order (NNLO) threshold corrections to heavy quark production at NNLL accuracy are also studied 
and the difference between single-particle inclusive (1PI) and pair inclusive (PIM) kinematics is highlighted. Similar NNLO expansions have recently been presented for electroweak-boson [20, 21], direct photon [22, 23], and jet [24] hadroproduction.

In the next section we briefly review the resummation formalism and give the expression for the resummed cross section in moment space. In Section 3 we present NLO and NNLO expansions at NNLL accuracy in single-particle inclusive kinematics and we discuss in detail the role of subleading logarithms. We also present numerical results for the top total cross section and transverse momentum distributions at the Tevatron. In Section 4 we present the next-tonext-to-next-to-leading order $\left(\mathrm{N}^{3} \mathrm{LO}\right)$ corrections at NNLL accuracy, and again we study the role of subleading logarithms at that order. In Section 5 we discuss $\mathrm{N}^{4} \mathrm{LO}$ and higher-order corrections. In Appendix A we list several formulas for the Mellin transforms that are used in the calculations. In Appendix B we collect some formulas for the integrations involved in the calculation of the hadronic total and differential cross sections. In Appendix $\mathrm{C}$ we present results for the NLO and NNLO expansions in PIM kinematics.

\section{Resummed cross section}

We begin by briefly reviewing the resummation formalism of Refs. [6, 7, 11]. We will mostly discuss the single-particle inclusive cross section but will make a few comments on pair inclusive kinematics where appropriate. The differences between the two kinematics in the expression for the resummed cross section are minimal.

The factorized single-heavy quark inclusive cross section for hadron-hadron collisions,

$$
h_{A}\left(p_{A}\right)+h_{B}\left(p_{B}\right) \rightarrow Q\left(p_{1}\right)+X[\bar{Q}],
$$

where the $h$ 's are the colliding hadrons, $Q$ is the produced heavy quark, and $X$ represents the additional partons in the final state including the heavy antiquark, takes the form of a convolution of the perturbative short-distance cross section $\hat{\sigma}$ with the universal parton distribution functions $\phi$ :

$$
\sigma_{h_{A} h_{B} \rightarrow Q X}=\int d x_{a} d x_{b} \phi_{f_{a} / h_{A}}\left(x_{a}, \mu_{F}^{2}\right) \phi_{f_{b} / h_{B}}\left(x_{b}, \mu_{F}^{2}\right) \hat{\sigma}_{f_{a} f_{b} \rightarrow Q X}\left(s_{4}, t_{1}, u_{1}, m^{2}, \mu_{F}^{2}, \alpha_{s}\left(\mu_{R}^{2}\right)\right),
$$

where $\mu_{F}$ and $\mu_{R}$ are the factorization and renormalization scales, respectively. Note that here and in the following $\sigma$ and $\hat{\sigma}$ can denote either the total cross section or any relevant differential cross section. The parton processes involved at the Born level are

$$
\begin{aligned}
& q\left(p_{a}\right)+\bar{q}\left(p_{b}\right) \rightarrow Q\left(p_{1}\right)+\bar{Q}\left(p_{2}\right), \\
& g\left(p_{a}\right)+g\left(p_{b}\right) \rightarrow Q\left(p_{1}\right)+\bar{Q}\left(p_{2}\right) .
\end{aligned}
$$

The $q \bar{q}$ channel is dominant for top production at the Tevatron and contributes over $90 \%$ of the cross section at the Born level. The partonic invariants in Eq. (2.2) are defined by

$$
s=\left(p_{a}+p_{b}\right)^{2}, \quad t_{1}=\left(p_{a}-p_{1}\right)^{2}-m^{2}, \quad u_{1}=\left(p_{b}-p_{1}\right)^{2}-m^{2},
$$

with $m$ the heavy quark mass, while $s_{4}=s+t_{1}+u_{1}$ measures the distance from threshold; at threshold $s_{4}=0$. 
By taking moments and replacing the incoming hadrons by partons, the convolution in Eq. (2.2) simplifies to a product [7, 11],

$$
\tilde{\sigma}_{f_{a} f_{b} \rightarrow Q X}(N)=\tilde{\phi}_{f_{a} / f_{a}}\left(N_{a}\right) \tilde{\phi}_{f_{b} / f_{b}}\left(N_{b}\right) \hat{\sigma}_{f_{a} f_{b} \rightarrow Q X}(N) .
$$

The moments are defined by $\hat{\sigma}(N)=\int\left(d s_{4} / s\right) e^{-N s_{4} / s} \hat{\sigma}\left(s_{4}\right)$, with $N$ the moment variable, and similarly for the $\phi_{i}$ 's with respect to $x_{i}$. The definition of $N_{i}$ depends on the kinematics. For 1PI kinematics, $N_{a}=N(-u / s)$ and $N_{b}=N(-t / s)$ [11]. For PIM kinematics, $N_{a}=N_{b}=N$ [7].

The short-distance perturbative cross section $\hat{\sigma}$ still displays sensitivity to soft gluon emission. The incomplete cancellation between graphs with gluon emission and virtual gluon corrections manifests itself in the occurence in $\hat{\sigma}$ of "plus" distributions which are singular at $s_{4}=0$, the partonic threshold. At $n$th order in $\alpha_{s}$ (beyond the Born term), these distributions are of the form $\left[\left(\ln ^{k}\left(s_{4} / m^{2}\right)\right) / s_{4}\right]_{+}, k \leq 2 n-1$. The leading logarithms correspond to $k=2 n-1$, NLL to $k=2 n-2$, NNLL to $k=2 n-3$ and so on. Under moments $\left[\left(\ln ^{2 n-1}\left(s_{4} / m^{2}\right)\right) / s_{4}\right]_{+} \rightarrow \ln ^{2 n} N$ (see Appendix A), and our goal becomes to resum logarithms of $N$.

To separate these soft gluon effects from the hard scattering, a refactorization is introduced [6, 7, 11, 16,

$$
\tilde{\sigma}_{f_{a} f_{b} \rightarrow Q X}(N)=\tilde{\psi}_{f_{a} / f_{a}}\left(N_{a}\right) \tilde{\psi}_{f_{b} / f_{b}}\left(N_{b}\right) H_{I J}^{f_{a} f_{b}} \tilde{S}_{J I}^{f_{a} f_{b}}\left(m /\left(N \mu_{F}\right)\right),
$$

where the $\psi$ 's are center-of-mass parton distributions [1] that absorb the universal collinear singularities associated with the initial-state partons, and $S$ is the soft-gluon function that describes non-collinear soft gluon emission. The mass of the heavy quarks protects the final state from collinear singularities. $H$ is the hard-scattering function and is free of soft-gluon effects and thus independent of $N . H$ and $S$ are matrices in the space of color exchanges $(I, J$ are color indices) and differ for each partonic process.

Using Eqs. (2.5) and (2.6) to solve for the perturbative cross section $\hat{\sigma}$, we then have $\hat{\sigma}(N)=\left(\tilde{\psi}_{f_{a} / f_{a}} \tilde{\psi}_{f_{b} / f_{b}} /\left(\tilde{\phi}_{f_{a} / f_{a}} \tilde{\phi}_{f_{b} / f_{b}}\right)\right) \operatorname{Tr}[H \tilde{S}]$. After resumming the $N$-dependence in $\psi / \phi$ and $S$ [6, 7, 11], we obtain the resummed heavy quark cross section at NLL accuracy in moment space:

$$
\begin{gathered}
\hat{\sigma}_{f_{a} f_{b} \rightarrow Q X}(N)=\exp \left[E^{\left(f_{a}\right)}\left(N_{a}\right)+E^{\left(f_{b}\right)}\left(N_{b}\right)\right] \exp \left[2 \int_{\mu_{F}}^{m} \frac{d \mu^{\prime}}{\mu^{\prime}}\left(\gamma_{a}\left(\alpha_{s}\left(\mu^{\prime 2}\right)\right)+\gamma_{b}\left(\alpha_{s}\left(\mu^{\prime 2}\right)\right)\right)\right] \\
\times \exp \left[4 \int_{\mu_{R}}^{m} \frac{d \mu^{\prime}}{\mu^{\prime}} \beta\left(\alpha_{s}\left(\mu^{\prime 2}\right)\right)\right] \operatorname{Tr}\left\{H^{f_{a} f_{b}}\left(\alpha_{s}\left(\mu_{R}^{2}\right)\right)\right. \\
\left.\times \bar{P} \exp \left[\int_{m}^{m / N} \frac{d \mu^{\prime}}{\mu^{\prime}}\left(\Gamma_{S}^{f_{a} f_{b}}\right)^{\dagger}\left(\alpha_{s}\left(\mu^{\prime 2}\right)\right)\right] \tilde{S}^{f_{a} f_{b}}\left(1, \alpha_{s}\left(m^{2} / N^{2}\right)\right) P \exp \left[\int_{m}^{m / N} \frac{d \mu^{\prime}}{\mu^{\prime}} \Gamma_{S}^{f_{a} f_{b}}\left(\alpha_{s}\left(\mu^{\prime 2}\right)\right)\right]\right\} .
\end{gathered}
$$

This expression is actually valid for both 1PI and PIM kinematics with appropriate definitions for $N_{a}$ and $N_{b}$ as discussed previously.

The first exponent in Eq. (2.7) resums the $N$-dependence of the ratio $\tilde{\psi}_{f_{i} / f_{i}} / \tilde{\phi}_{f_{i} / f_{i}}$ and is given in the $\overline{\mathrm{MS}}$ scheme by

$$
E^{\left(f_{i}\right)}\left(N_{i}\right)=-\int_{0}^{1} d z \frac{z^{N_{i}-1}-1}{1-z}\left\{\int_{(1-z)^{2} s}^{\mu_{F}^{2}} \frac{d \mu^{\prime 2}}{\mu^{\prime 2}} A^{\left(f_{i}\right)}\left[\alpha_{s}\left(\mu^{\prime 2}\right)\right]+\frac{1}{2} \kappa^{\left(f_{i}\right)}\left[\alpha_{s}\left((1-z)^{2} s\right)\right]\right\} .
$$

${ }^{1}$ Note that although we formally have NLL accuracy in the resummed exponent, after matching with the exact NLO cross section we can determine all NNLL terms in the finite-order expansions. 
At next-to-leading order accuracy in $\ln N$, we need $A^{(f)}$ at two-loops, $A^{(f)}\left(\alpha_{s}\right)=C_{f}\left[\alpha_{s} / \pi\right.$ $\left.+\left(\alpha_{s} / \pi\right)^{2} K / 2\right]$, and $\kappa^{(f)}=2 C_{f}\left(\alpha_{s} / \pi\right)\left(1-\ln \left(2 \nu_{f}\right)\right)$. Here $C_{f}=C_{F}=\left(N_{c}^{2}-1\right) /\left(2 N_{c}\right)$ for an incoming quark, and $C_{f}=C_{A}=N_{c}$ for an incoming gluon, with $N_{c}$ the number of colors, while $K=C_{A}\left(67 / 18-\pi^{2} / 6\right)-5 n_{f} / 9$, where $n_{f}$ is the number of quark flavors. The $\nu_{i}$ terms are gauge dependent. They are defined by $\nu_{i} \equiv\left(\beta_{i} \cdot n\right)^{2} /|n|^{2}$, where $\beta_{i}=p_{i} \sqrt{2 / s}$ are the particle velocities and $n$ is the axial gauge vector. We note that all gauge dependence cancels out in the cross section.

In the DIS scheme, which is usually only applied to quarks, the first exponent in Eq. (2.7) is given in terms of the $\overline{\mathrm{MS}}$ result as

$$
\left.E^{(q)}\left(N_{i}\right)\right|_{\mathrm{DIS}}=\left.E^{(q)}\left(N_{i}\right)\right|_{\overline{\mathrm{MS}}}-\int_{0}^{1} d z \frac{z^{N_{i}-1}-1}{1-z}\left\{\int_{1}^{1-z} \frac{d \lambda}{\lambda} A^{(q)}\left[\alpha_{s}(\lambda s)\right]+B^{(q)}\left[\alpha_{s}((1-z) s)\right]\right\},
$$

where $B^{(q)}\left(\alpha_{s}\right)=-\left(3 C_{F} / 4\right)\left(\alpha_{s} / \pi\right)$.

The $\gamma_{a}$ are anomalous dimensions of the fields $\psi$ and are given at one loop by $\gamma_{q}=$ $\left(\alpha_{s} / \pi\right)\left(3 C_{F} / 4\right)$ and $\gamma_{g}=\left(\alpha_{s} / \pi\right)\left(\beta_{0} / 4\right)$ for quarks and gluons, respectively. The $\beta$ function is given by $\beta\left(\alpha_{s}\right) \equiv \mu d \ln g / d \mu=-\beta_{0} \alpha_{s} /(4 \pi)+\ldots$, with $\beta_{0}=\left(11 C_{A}-2 n_{f}\right) / 3$.

The trace appearing in the resummed expression is taken in color space. The symbols $P$ and $\bar{P}$ denote path ordering in the same sense as the variable $\mu^{\prime}$ and against it, respectively. The evolution of the soft function from scale $m / N$ to $m$ follows from its renormalization group properties and is given in terms of the soft anomalous dimension matrix $\Gamma_{S}$ [7]. For the determination of $\Gamma_{S}$ an appropriate choice of color basis has to be made. For the $q \bar{q}$ channel we use an $s$-channel singlet-octet basis, while for the $g g$ channel we use a basis consisting of three color tensors [7]. The soft anomalous dimension matrix, evaluated through the calculation of one-loop eikonal vertex corrections, has been presented for the partonic processes in heavy quark production in Refs. [6, 7, 13]. In the color bases that we use, the soft matrices, $S$, are diagonal for both partonic channels, and the hard matrix for the $q \bar{q}$ channel has only one non-zero element. At lowest order, the trace of the product of the hard and soft matrices reproduces the Born cross section in each partonic channel. We also note that the $\Gamma_{S}$ matrices are not diagonal in the color bases that we use. If we perform a diagonalization so that the $\Gamma_{S}$ matrices do become diagonal, then the path-ordered exponentials of matrices in the resummed expression reduce to simple exponentials; however, this diagonalization procedure is complicated in practice [13].

The integrations over $z$ in the exponents of Eqs. (2.7), (2.8) run over the region where the running coupling constant $\alpha_{s}$ diverges. The prescriptions of Refs. [3, 4, 5] have been proposed to avoid these soft gluon divergences in the resummed cross section. However, if we expand the exponents in the resummed cross section at fixed order in $\alpha_{s}$ and invert back to momentum space using the equations in Appendix A, no divergences are encountered and thus no prescription is required. In addition to avoiding the necessity for a resummation prescription, a finite-order expansion bypasses the need for the diagonalization procedure that we mentioned above, as well.

\section{$3 \quad$ NLO and NNLO threshold corrections}

In this section we expand the resummed cross section to next-to-leading and next-to-next-toleading orders. In the following $\sigma^{(n)}$ stands for the $n$ th-order differential corrections. Nominally, 
it denotes $s^{2} d^{2} \sigma^{(n)} /\left(d t_{1} d u_{1}\right)$ but it can also denote any other relevant differential cross section, such as $d^{2} \sigma^{(n)} /\left(d p_{T}^{2} d s_{4}\right)$, with $p_{T}$ the transverse momentum, or $d^{2} \sigma^{(n)} /\left(d y d s_{4}\right)$, with $y$ the rapidity, or $d^{2} \sigma^{(n)} /\left(d p_{T} d y\right)$, with appropriate Jacobians inserted into the definition of the Born term, $\sigma^{B}$, and the function $B_{\mathrm{QED}}$ for the $g g$ channel in the expressions below.

\subsection{NLO threshold corrections}

We first expand the resummed cross section to next-to-leading order in 1PI kinematics. These expansions are already known for both 1PI and PIM kinematics [7, 11, 13].

For the $q \bar{q}$ channel in the $\overline{\mathrm{MS}}$ scheme, the full next-to-leading-order threshold corrections are

$$
\begin{aligned}
\hat{\sigma}_{q \bar{q} \rightarrow Q \bar{Q}}^{\overline{\mathrm{MS}}(1)}\left(s_{4},\right. & \left.m^{2}, s, t_{1}, u_{1}, \mu_{F}, \mu_{R}\right)=\sigma_{q \bar{q} \rightarrow Q \bar{Q}}^{B} \frac{\alpha_{s}\left(\mu_{R}^{2}\right)}{\pi}\left\{4 C_{F}\left[\frac{\ln \left(s_{4} / m^{2}\right)}{s_{4}}\right]_{+}\right. \\
+ & {\left.\left[\frac{1}{s_{4}}\right]_{+}\left[2 \operatorname{Re} \Gamma_{22}^{\prime q \bar{q}}-2 C_{F}+2 C_{F} \ln \left(\frac{s m^{2}}{t_{1} u_{1}}\right)-2 C_{F} \ln \left(\frac{\mu_{F}^{2}}{m^{2}}\right)\right]\right\} } \\
+ & \delta\left(s_{4}\right) \sigma_{\overline{\mathrm{MS}}}^{(1) q \bar{q} \mathrm{~S}+\mathrm{V}},
\end{aligned}
$$

where $\sigma_{\overline{\mathrm{MS}}}^{(1) q \overline{\mathrm{S}} \mathrm{S}+\mathrm{V}}$ denotes the soft plus virtual $(S+V) \delta\left(s_{4}\right)$ terms in the NLO cross section that can be obtained from Eq. (4.7) in Ref. [25] (with $t_{1}$ and $u_{1}$ interchanged because of different definitions in that reference). Also

$$
\operatorname{Re}_{22}^{\prime q \bar{q}}=C_{F}\left[4 \ln \left(\frac{u_{1}}{t_{1}}\right)-\operatorname{Re} L_{\beta}\right]+\frac{C_{A}}{2}\left[-3 \ln \left(\frac{u_{1}}{t_{1}}\right)-\ln \left(\frac{m^{2} s}{t_{1} u_{1}}\right)+\operatorname{Re} L_{\beta}\right]
$$

is obtained from the real part of the one-loop soft anomalous dimension matrix element $\Gamma_{22}^{q \bar{q}}$ after dropping all gauge dependent terms and an overall coefficient $\alpha_{s} / \pi$. Here $L_{\beta}=(1-$ $\left.2 m^{2} / s\right) / \beta \cdot[\ln ((1-\beta) /(1+\beta))+\pi i]$, with $\beta=\sqrt{1-4 m^{2} / s}$, is the velocity-dependent eikonal function. The Born term is given by

$$
\sigma_{q \bar{q} \rightarrow Q \bar{Q}}^{B}=\pi \alpha_{s}^{2}\left(\mu_{R}^{2}\right) K_{q \bar{q}} N_{c} C_{F}\left[\frac{t_{1}^{2}+u_{1}^{2}}{s^{2}}+\frac{2 m^{2}}{s}\right]
$$

where $K_{q \bar{q}}=N_{c}^{-2}$ is a color average factor. In our calculation for the expansion, we used the result for the soft matrix at lowest order in Eq. (A5) of Ref. [24]. The lowest-order hard matrix has only one non-zero element, given by $H_{22}^{q \bar{q} \rightarrow Q \bar{Q}}=\left[2 /\left(N_{c} C_{F}\right)\right] \sigma_{q \bar{q} \rightarrow Q \bar{Q}}^{B}$.

To be sure, the expansion of the NLL resummed cross section does not give all the $\delta\left(s_{4}\right)$ terms, only those $\delta\left(s_{4}\right)$ terms involving the scale; these terms are $\sigma_{q \bar{q} \rightarrow Q \bar{Q}}^{B}\left(\alpha_{s}\left(\mu_{R}^{2}\right) / \pi\right) \delta\left(s_{4}\right)[(-3 / 2$ $\left.\left.+\ln \left(t_{1} u_{1} / m^{4}\right)\right) C_{F} \ln \left(\mu_{F}^{2} / m^{2}\right)+\left(\beta_{0} / 2\right) \ln \left(\mu_{R}^{2} / m^{2}\right)\right]$. The rest are obtained by simply matching with the NLO cross section in [25]. Thus we obtain all the $S+V$ terms at NLO. As shown in Refs. [26, 27] these terms dominate the cross section and are an excellent approximation at the partonic level to the exact NLO cross section close to threshold and even quite far from it. We note that the exact NLO cross section is the sum of the full $S+V$ terms and hard gluon corrections; the latter are not taken into account by threshold studies and vanish at threshold. 
In the DIS scheme, the corresponding result for the $q \bar{q}$ channel is

$$
\begin{aligned}
& \hat{\sigma}_{q \bar{q} \rightarrow Q \bar{Q}}^{\mathrm{DIS}(1)}\left(s_{4},\right.\left.m^{2}, s, t_{1}, u_{1}, \mu_{F}, \mu_{R}\right)=\sigma_{q \bar{q} \rightarrow Q \bar{Q}}^{B} \frac{\alpha_{s}\left(\mu_{R}^{2}\right)}{\pi}\left\{2 C_{F}\left[\frac{\ln \left(s_{4} / m^{2}\right)}{s_{4}}\right]_{+}\right. \\
&\left.+\left[\frac{1}{s_{4}}\right]_{+}\left[2 \operatorname{Re} \Gamma_{22}^{\prime q \bar{q}}-\frac{C_{F}}{2}+C_{F} \ln \left(\frac{s^{2}}{t_{1} u_{1}}\right)-2 C_{F} \ln \left(\frac{\mu_{F}^{2}}{m^{2}}\right)\right]\right\} \\
&+\delta\left(s_{4}\right) \sigma_{\mathrm{DIS}}^{(1) q \overline{\mathrm{S}}+\mathrm{V}},
\end{aligned}
$$

where $\sigma_{\mathrm{DIS}}^{(1) q \bar{q} \mathrm{~S}+\mathrm{V}}$ can be obtained from Eq. (4.14) in 25.

For the $g g$ channel in the $\overline{\mathrm{MS}}$ scheme the NLO threshold corrections are given by

$$
\begin{gathered}
\hat{\sigma}_{g g \rightarrow Q \bar{Q}}^{\overline{\mathrm{MS}}(1)}\left(s_{4}, m^{2}, s, t_{1}, u_{1}, \mu_{F}, \mu_{R}\right)=\sigma_{g g \rightarrow Q \bar{Q}}^{B} \frac{\alpha_{s}\left(\mu_{R}^{2}\right)}{\pi}\left\{4 C_{A}\left[\frac{\ln \left(s_{4} / m^{2}\right)}{s_{4}}\right]_{+}-2 C_{A} \ln \left(\frac{\mu_{F}^{2}}{m^{2}}\right)\left[\frac{1}{s_{4}}\right]_{+}\right\} \\
+\alpha_{s}^{3}\left(\mu_{R}^{2}\right) K_{g g} B_{Q E D}\left[\frac{1}{s_{4}}\right]_{+}\left\{N _ { c } ( N _ { c } ^ { 2 } - 1 ) \frac { ( t _ { 1 } ^ { 2 } + u _ { 1 } ^ { 2 } ) } { s ^ { 2 } } \left[\left(-C_{F}+\frac{C_{A}}{2}\right) \operatorname{Re} L_{\beta}\right.\right. \\
\left.+\frac{C_{A}}{2} \ln \left(\frac{m^{2} s}{t_{1} u_{1}}\right)-C_{F}\right]+\frac{\left(N_{c}^{2}-1\right)}{N_{c}}\left(C_{F}-C_{A}\right) \operatorname{Re} L_{\beta} \\
\left.+C_{F} \frac{\left(N_{c}^{2}-1\right)}{N_{c}}+\frac{N_{c}^{2}}{2}\left(N_{c}^{2}-1\right) \ln \left(\frac{u_{1}}{t_{1}}\right) \frac{\left(t_{1}^{2}-u_{1}^{2}\right)}{s^{2}}\right\}+\delta\left(s_{4}\right) \sigma_{\frac{\mathrm{MS}}{(1) g \mathrm{~S}+\mathrm{V}},}
\end{gathered}
$$

where $K_{g g}=\left(N_{c}^{2}-1\right)^{-2}$ is a color average factor, and

$$
B_{\mathrm{QED}}=\frac{t_{1}}{u_{1}}+\frac{u_{1}}{t_{1}}+\frac{4 m^{2} s}{t_{1} u_{1}}\left(1-\frac{m^{2} s}{t_{1} u_{1}}\right) .
$$

Here, $\sigma_{\overline{\mathrm{MS}}}^{(1) g g \mathrm{~S}+\mathrm{V}}$ again denotes the soft plus virtual $\delta\left(s_{4}\right)$ terms in the NLO cross section. These terms are given by Eq. (6.19) in Ref. [28]; we note, however, that in that reference the scale was set equal to $m$, therefore in addition to those terms we have to include in $\sigma_{\overline{\mathrm{MS}}}^{(1) g g \mathrm{~S}+\mathrm{V}}$ the terms $\sigma_{g g \rightarrow Q \bar{Q}}^{B}\left(\alpha_{s}\left(\mu_{R}^{2}\right) / \pi\right)\left[C_{A} \ln \left(t_{1} u_{1} / m^{4}\right) \ln \left(\mu_{F}^{2} / m^{2}\right)+\left(\beta_{0} / 2\right) \ln \left(\mu_{R}^{2} / \mu_{F}^{2}\right)\right]$. The Born term is given by

$$
\sigma_{g g \rightarrow Q \bar{Q}}^{B}=2 \pi \alpha_{s}^{2}\left(\mu_{R}^{2}\right) K_{g g} N_{c} C_{F}\left[C_{F}-C_{A} \frac{t_{1} u_{1}}{s^{2}}\right] B_{\mathrm{QED}}
$$

In our calculation for the expansion we used the result for the soft matrix at lowest order in Eq. (C3) of Ref. [24]. The lowest-order hard matrix has the form of Eq. (C6) of Ref. [24], with independent elements $H_{11}^{g g \rightarrow Q \bar{Q}}=\pi \alpha_{s}^{2} B_{\mathrm{QED}} K_{g g} /\left(2 N_{c}^{2}\right), H_{13}^{g g \rightarrow Q \bar{Q}}=N_{c} H_{11}^{g g \rightarrow Q \bar{Q}}\left(t_{1}^{2}-u_{1}^{2}\right) / s^{2}$, and $H_{33}^{g g \rightarrow Q \bar{Q}}=N_{c}^{2} H_{11}^{g g \rightarrow Q \bar{Q}}\left(1-4 t_{1} u_{1} / s^{2}\right)$.

Again as shown in Refs. [26, 27] these corrections dominate the cross section near threshold and are an excellent approximation at the partonic level to the exact NLO cross section. For NLO expansions in PIM kinematics see Appendix C.

\subsection{NNLO-NNLL threshold corrections for $q \bar{q} \rightarrow Q \bar{Q}$}

Next we derive the NNLO-NNLL threshold corrections from the two-loop expansion of the resummed cross section. 
For the $q \bar{q}$ channel in the $\overline{\mathrm{MS}}$ scheme these corrections are

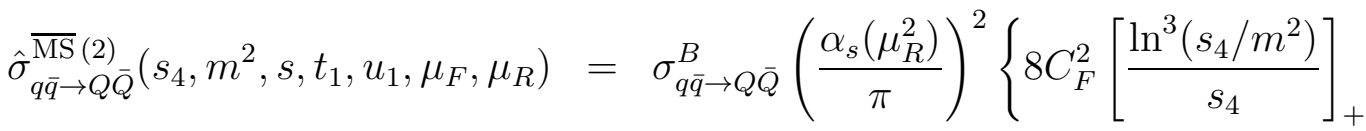

$$
\begin{aligned}
& +\left[\frac{\ln ^{2}\left(s_{4} / m^{2}\right)}{s_{4}}\right]_{+}\left[-\beta_{0} C_{F}+12 C_{F}\left(\operatorname{Re}_{22}^{\prime q \bar{q}}-C_{F}+C_{F} \ln \left(\frac{s m^{2}}{t_{1} u_{1}}\right)-C_{F} \ln \left(\frac{\mu_{F}^{2}}{m^{2}}\right)\right)\right] \\
& +\left[\frac{\ln \left(s_{4} / m^{2}\right)}{s_{4}}\right]_{+}\left[4\left[\operatorname{Re} \Gamma_{22}^{q \bar{q}}-C_{F}-C_{F} \ln \left(\frac{t_{1} u_{1}}{s m^{2}}\right)-C_{F} \ln \left(\frac{\mu_{F}^{2}}{m^{2}}\right)\right]^{2}\right. \\
& +4 \Gamma_{12}^{q \bar{q}} \Gamma_{21}^{\prime q \bar{q}}-\beta_{0}\left[\operatorname{Re}_{22}^{\prime q \bar{q}}-C_{F}-C_{F} \ln \left(\frac{t_{1} u_{1}}{s m^{2}}\right)-C_{F} \ln \left(\frac{\mu_{R}^{2}}{m^{2}}\right)\right] \\
& \left.\left.+2 C_{F} K-16 \zeta_{2} C_{F}^{2}+4 C_{F} c \frac{(1) q \bar{q} \mathrm{~S}+\mathrm{V}}{\mathrm{MS}}\right]\right\}+\mathcal{O}\left(\left[\frac{1}{s_{4}}\right]_{+}\right),
\end{aligned}
$$

where $c\left(\frac{1)}{\mathrm{MS}} \bar{q} \mathrm{~S}+\mathrm{V}\right.$ is defined by

$$
\sigma_{\overline{\mathrm{MS}}}^{(1) q \overline{\mathrm{q}} \mathrm{S}+\mathrm{V}}=\frac{\alpha_{s}}{\pi} \sigma_{q \bar{q} \rightarrow Q \bar{Q}}^{B} c \frac{(1) q \overline{\mathrm{MS}}}{\mathrm{S}} \mathrm{V}^{\mathrm{V}},
$$

and the off-diagonal elements of the soft anomalous dimension matrix (dropping an overall $\left.\alpha_{s} / \pi\right)$ are

$$
\Gamma_{21}^{\prime q \bar{q}}=2 \ln \left(\frac{u_{1}}{t_{1}}\right), \quad \Gamma_{12}^{\prime q \bar{q}}=\frac{C_{F}}{C_{A}} \ln \left(\frac{u_{1}}{t_{1}}\right) .
$$

We are able to derive all the NNLL terms by matching with the $S+V$ terms in the NLO cross section, Eq. (3.9).

In addition, we can derive at NNLL accuracy the following $\left[1 / s_{4}\right]_{+}$and $\delta\left(s_{4}\right)$ terms involving logarithms of the factorization and renormalization scales:

$$
\begin{aligned}
& \sigma_{q \bar{q} \rightarrow Q \bar{Q}}^{B}\left(\frac{\alpha_{s}\left(\mu_{R}^{2}\right)}{\pi}\right)^{2}\left[\frac{1}{s_{4}}\right]_{+}\left\{\ln ^{2}\left(\frac{\mu_{F}^{2}}{m^{2}}\right) C_{F}\left[C_{F}\left(3-2 \ln \left(\frac{t_{1} u_{1}}{m^{4}}\right)\right)+\frac{\beta_{0}}{4}\right]\right. \\
& -\frac{3}{2} C_{F} \beta_{0} \ln \left(\frac{\mu_{R}^{2}}{m^{2}}\right) \ln \left(\frac{\mu_{F}^{2}}{m^{2}}\right)+\ln \left(\frac{\mu_{F}^{2}}{m^{2}}\right)\left[-2 C_{F} \hat{T}_{\overline{\mathrm{MS}}}^{(1) q \bar{q}}-C_{F} K+8 C_{F}^{2} \zeta_{2}\right. \\
& \left.+C_{F}\left(2 \ln \left(\frac{t_{1} u_{1}}{m^{4}}\right)-3\right)\left(\operatorname{Re}_{22}^{\prime q \bar{q}}-C_{F}+C_{F} \ln \left(\frac{s m^{2}}{t_{1} u_{1}}\right)\right)\right] \\
& \left.+\frac{3}{2} \beta_{0} \ln \left(\frac{\mu_{R}^{2}}{m^{2}}\right)\left[\operatorname{Re} \Gamma_{22}^{q \bar{q}}-C_{F}-C_{F} \ln \left(\frac{t_{1} u_{1}}{m^{2} s}\right)\right]\right\} \\
& +\sigma_{q \bar{q} \rightarrow Q \bar{Q}}^{B}\left(\frac{\alpha_{s}\left(\mu_{R}^{2}\right)}{\pi}\right)^{2} \delta\left(s_{4}\right)\left\{\operatorname { l n } ^ { 2 } ( \frac { \mu _ { F } ^ { 2 } } { m ^ { 2 } } ) \left[\frac{1}{2} C_{F}^{2} \ln ^{2}\left(\frac{t_{1} u_{1}}{m^{4}}\right)+\frac{9}{8} C_{F}^{2}-\frac{3}{2} C_{F}^{2} \ln \left(\frac{t_{1} u_{1}}{m^{4}}\right)\right.\right. \\
& \left.-2 C_{F}^{2} \zeta_{2}-\frac{\beta_{0}}{8} C_{F} \ln \left(\frac{t_{1} u_{1}}{m^{4}}\right)+\frac{3}{16} \beta_{0} C_{F}\right]+\frac{3}{16} \beta_{0}^{2} \ln ^{2}\left(\frac{\mu_{R}^{2}}{m^{2}}\right) \\
& \left.+\frac{3}{4} C_{F} \beta_{0} \ln \left(\frac{\mu_{F}^{2}}{m^{2}}\right) \ln \left(\frac{\mu_{R}^{2}}{m^{2}}\right)\left[\ln \left(\frac{t_{1} u_{1}}{m^{4}}\right)-\frac{3}{2}\right]\right\},
\end{aligned}
$$

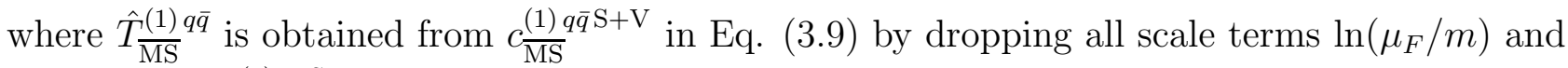

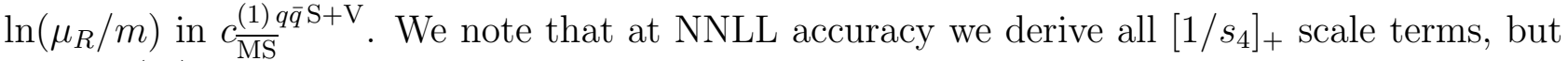
in the $\delta\left(s_{4}\right)$ coefficient we can only determine quadratic terms in the scale logarithms. 


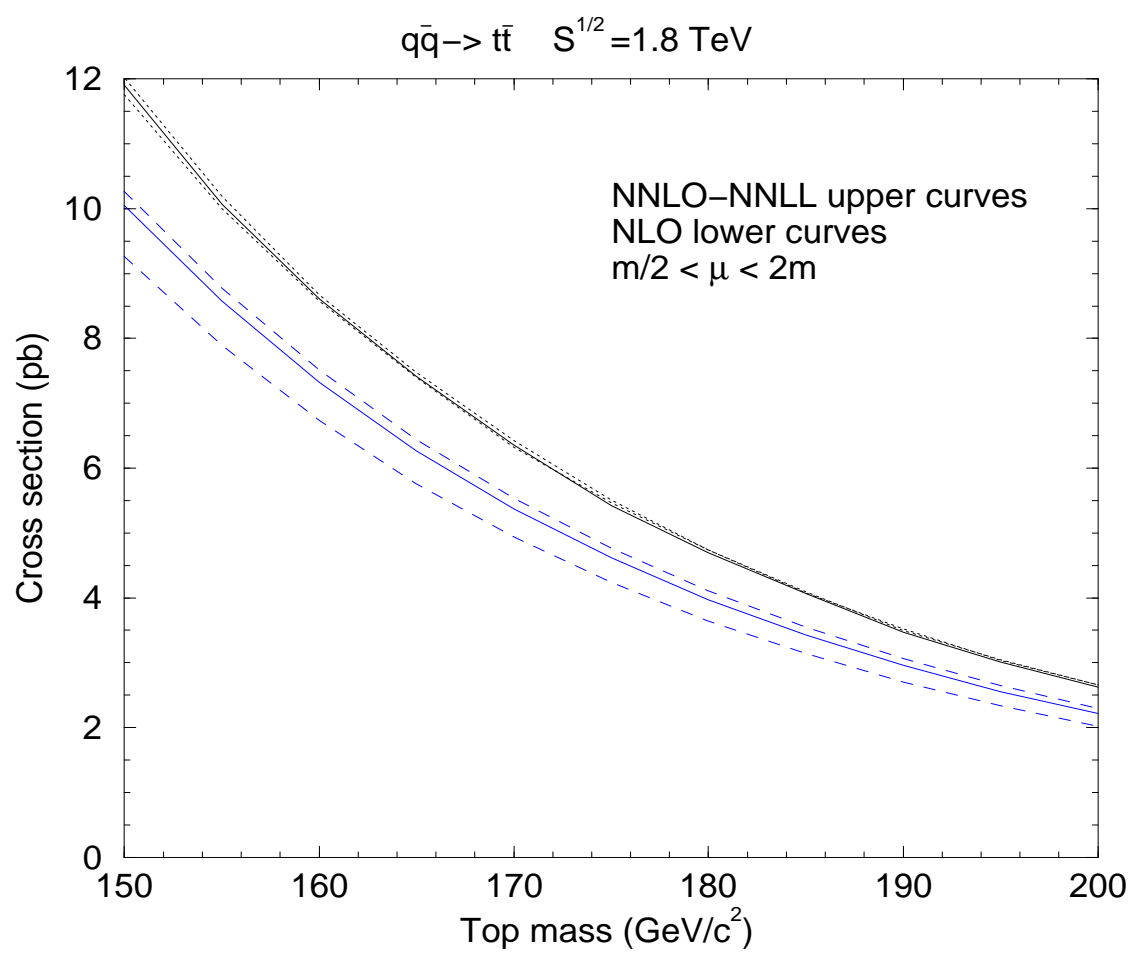

Fig. 1. Top quark production at the Tevatron with $\sqrt{S}=1.8 \mathrm{TeV}$ for the $q \bar{q} \rightarrow t \bar{t}$ channel in the $\overline{\mathrm{MS}}$ scheme. Plotted are the exact NLO cross section for $\mu=m$ (lower solid line), $m / 2$ and $2 m$ (upper and lower dashed lines), and the NNLL-NNLO cross section for $\mu=m$ (upper solid line), $m / 2$ and $2 m$ (upper and lower dotted lines). 


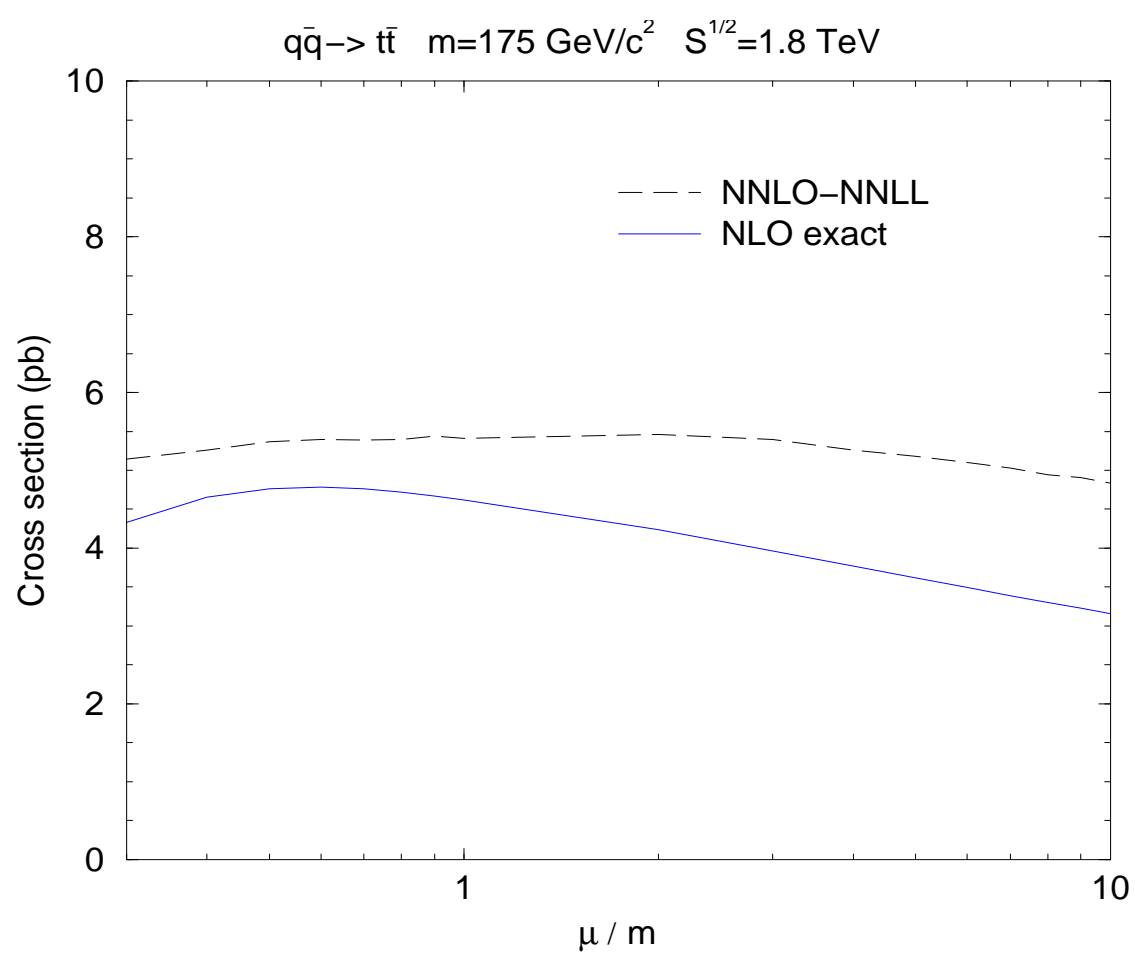

Fig. 2. The scale dependence of the cross section for $q \bar{q} \rightarrow t \bar{t}$ in the $\overline{\mathrm{MS}}$ scheme at the Tevatron with $\sqrt{S}=1.8 \mathrm{TeV}$ and $m=175 \mathrm{GeV} / c^{2}$.

Since the $q \bar{q}$ channel is dominant for top quark production at the Tevatron, it is worthwhile to show some numerical results for the hadronic cross section in that channel alone before presenting the full cross section (for details of the hadronic calculation see Appendix B). In Fig. 1 we plot the NNLO-NNLL top quark cross section $q \bar{q} \rightarrow t \bar{t}$ at the Tevatron with $\sqrt{S}=1.8$ $\mathrm{TeV}$ together with the exact NLO cross section [25, 29] as a function of the top mass; we have set $\mu \equiv \mu_{F}=\mu_{R}$. Here and in the rest of the paper we use the CTEQ5M parton densities [30] when calculating $\overline{\mathrm{MS}}$ results. We note the significant increase of the cross section at NNLO along with the dramatic reduction in scale variation between $\mathrm{m} / 2$ and $2 \mathrm{~m}$. This reduction is also evident for a wide range of scale choices in Fig. 2. The NNLO cross section is larger than at NLO and relatively flat with respect to scale variations.

In Table 1 we present detailed numerical results for the $q \bar{q}$ corrections to the top quark cross section at the Tevatron with $m=175 \mathrm{GeV} / c^{2}$ through NNLO at both NLL and NNLL accuracy. Some of these numbers will be useful in our discussion in the next subsection.

The NNLO-NNLL threshold corrections in the DIS scheme are

$$
\begin{aligned}
\hat{\sigma}_{q \bar{q} \rightarrow Q \bar{Q}}^{\operatorname{DIS}(2)} & \left(s_{4}, m^{2}, s, t_{1}, u_{1}, \mu_{F}, \mu_{R}\right)=\sigma_{q \bar{q} \rightarrow Q \bar{Q}}^{B}\left(\frac{\alpha_{s}\left(\mu_{R}^{2}\right)}{\pi}\right)^{2}\left\{2 C_{F}^{2}\left[\frac{\ln ^{3}\left(s_{4} / m^{2}\right)}{s_{4}}\right]_{+}\right. \\
& +\left[\frac{\ln ^{2}\left(s_{4} / m^{2}\right)}{s_{4}}\right]_{+}\left[-\frac{3 \beta_{0}}{4} C_{F}+6 C_{F}\left(\operatorname{Re}_{22}^{\prime q \bar{q}}-\frac{C_{F}}{4}+\frac{C_{F}}{2} \ln \left(\frac{s^{2}}{t_{1} u_{1}}\right)-C_{F} \ln \left(\frac{\mu_{F}^{2}}{m^{2}}\right)\right)\right. \\
& +\left[\frac{\ln \left(s_{4} / m^{2}\right)}{s_{4}}\right]_{+}\left[4\left[\operatorname{Re}_{22}^{\prime q \bar{q}}-\frac{C_{F}}{4}-\frac{C_{F}}{2} \ln \left(\frac{t_{1} u_{1}}{s^{2}}\right)-C_{F} \ln \left(\frac{\mu_{F}^{2}}{m^{2}}\right)\right]^{2}\right.
\end{aligned}
$$




\begin{tabular}{|c|c|c|c|}
\hline$q \bar{q} \rightarrow t \bar{t}$ & $\mu=m$ & $\mu=m / 2$ & $\mu=2 m$ \\
\hline Born & 3.81 & 5.30 & 2.87 \\
\hline NLO-exact & 0.81 & -0.53 & 1.37 \\
\hline NLO-NLL & 1.31 & 0.03 & 1.81 \\
\hline NLO-full $S+V$ & 1.26 & -0.06 & 1.78 \\
\hline NNLO-NLL & 1.01 & 0.67 & 1.29 \\
\hline NNLO-NNLL & 0.80 & 0.62 & 1.22 \\
\hline
\end{tabular}

Table 1: The $\overline{\mathrm{MS}}$ corrections for top quark production in the $q \bar{q}$ channel in pb for $p \bar{p}$ collisions with $\sqrt{S}=1.8 \mathrm{TeV}$ and $m=175 \mathrm{GeV} / c^{2}$. Here $\mu=\mu_{F}=\mu_{R}$.

$$
\begin{aligned}
& +4 \Gamma_{12}^{\prime q \bar{q}} \Gamma_{21}^{\prime q \bar{q}}-\beta_{0}\left[\operatorname{Re} \Gamma_{22}^{\prime}-\frac{5}{8} C_{F}-\frac{3}{4} C_{F} \ln \left(\frac{t_{1} u_{1}}{m^{4}}\right)-\frac{1}{2} C_{F} \ln \left(\frac{\mu_{R}^{2}}{s}\right)\right] \\
& \left.\left.+C_{F} K-4 \zeta_{2} C_{F}^{2}+2 C_{F} c_{\mathrm{DIS}}^{(1) q \overline{\mathrm{S}} \mathrm{S}+\mathrm{V}}\right]\right\}+\mathcal{O}\left(\left[\frac{1}{s_{4}}\right]_{+}\right)
\end{aligned}
$$

where $c_{\mathrm{DIS}}^{(1) q \bar{q} \mathrm{~S}+\mathrm{V}}$ is defined in analogy to Eq. (3.9). As for the $\overline{\mathrm{MS}}$ corrections, we can also derive additional $\left[1 / s_{4}\right]_{+}$and $\delta\left(s_{4}\right)$ terms involving the scale in the DIS scheme. These terms are

$$
\begin{aligned}
& \sigma_{q \bar{q} \rightarrow Q \bar{Q}}^{B}\left(\frac{\alpha_{s}\left(\mu_{R}^{2}\right)}{\pi}\right)^{2}\left[\frac{1}{s_{4}}\right]_{+}\left\{\ln ^{2}\left(\frac{\mu_{F}^{2}}{m^{2}}\right) C_{F}\left[C_{F}\left(3-2 \ln \left(\frac{t_{1} u_{1}}{m^{4}}\right)\right)+\frac{\beta_{0}}{4}\right]\right. \\
& -\frac{3}{2} C_{F} \beta_{0} \ln \left(\frac{\mu_{R}^{2}}{m^{2}}\right) \ln \left(\frac{\mu_{F}^{2}}{m^{2}}\right)+\ln \left(\frac{\mu_{F}^{2}}{m^{2}}\right)\left[-2 C_{F} \hat{T}_{\mathrm{DIS}}^{(1) q \bar{q}}-C_{F} K+4 C_{F}^{2} \zeta_{2}\right. \\
& \left.+C_{F}\left(\ln \left(\frac{t_{1} u_{1}}{m^{4}}\right)-\frac{3}{2}\right)\left(2 \operatorname{Re} \Gamma_{22}^{\prime q \bar{q}}-\frac{C_{F}}{2}+C_{F} \ln \left(\frac{s^{2}}{t_{1} u_{1}}\right)\right)\right] \\
& \left.+\frac{3}{2} \beta_{0} \ln \left(\frac{\mu_{R}^{2}}{m^{2}}\right)\left[\operatorname{Re}_{22}^{\prime q \bar{q}}-\frac{C_{F}}{4}-\frac{C_{F}}{2} \ln \left(\frac{t_{1} u_{1}}{s^{2}}\right)\right]\right\} \\
& +\sigma_{q \bar{q} \rightarrow Q \bar{Q}}^{B}\left(\frac{\alpha_{s}\left(\mu_{R}^{2}\right)}{\pi}\right)^{2} \delta\left(s_{4}\right)\left\{\operatorname { l n } ^ { 2 } ( \frac { \mu _ { F } ^ { 2 } } { m ^ { 2 } } ) \left[\frac{1}{2} C_{F}^{2} \ln ^{2}\left(\frac{t_{1} u_{1}}{m^{4}}\right)+\frac{9}{8} C_{F}^{2}-\frac{3}{2} C_{F}^{2} \ln \left(\frac{t_{1} u_{1}}{m^{4}}\right)\right.\right. \\
& \left.-2 C_{F}^{2} \zeta_{2}-\frac{\beta_{0}}{8} C_{F} \ln \left(\frac{t_{1} u_{1}}{m^{4}}\right)+\frac{3}{16} \beta_{0} C_{F}\right]+\frac{3}{16} \beta_{0}^{2} \ln ^{2}\left(\frac{\mu_{R}^{2}}{m^{2}}\right) \\
& \left.+\frac{3}{4} C_{F} \beta_{0} \ln \left(\frac{\mu_{F}^{2}}{m^{2}}\right) \ln \left(\frac{\mu_{R}^{2}}{m^{2}}\right)\left[\ln \left(\frac{t_{1} u_{1}}{m^{4}}\right)-\frac{3}{2}\right]\right\}
\end{aligned}
$$

where $\hat{T}_{\mathrm{DIS}}^{(1) q \bar{q}}$ is defined in analogy to its $\overline{\mathrm{MS}}$ counterpart.

At the Tevatron, with $\sqrt{S}=1.8 \mathrm{TeV}$ and $\mu_{F}=\mu_{R}=m=175 \mathrm{GeV} / \mathrm{c}^{2}$, and with the CTEQ5D parton densities [30], the exact NLO cross section in the DIS scheme is $4.60 \mathrm{pb}$ and the NNLO-NNLL corrections provide an additional $0.30 \mathrm{pb}$. The NNLO corrections in the DIS scheme are much smaller than the corresponding $\overline{\mathrm{MS}}$ corrections; that follows from the definition of the two schemes.

Results for the NNLO expansion of the resummed cross section in PIM kinematics in both the $\overline{\mathrm{MS}}$ and DIS schemes are presented in Appendix C (see also Ref. [13]). The differences between the expansions in the two different kinematics are in the extra terms involving $\ln \left(t_{1} u_{1} / m^{4}\right)$ 
for the 1PI fixed-order expansions relative to the PIM expansions and in the matching terms needed to reach NNLL accuracy [19].

\subsection{Subleading logarithms and resummation prescriptions}

In the previous subsection we derived all the NNLO threshold corrections for $q \bar{q} \rightarrow t \bar{t}$ through NNLL accuracy. It is interesting to study the effect of subleading $\left[1 / s_{4}\right]_{+}$and $\delta\left(s_{4}\right)$ terms that come about when inverting the cross section from moment to momentum space. As we will see, this is intimately related to the disagreements between various resummation prescriptions that have been proposed. These prescriptions are needed to avoid the soft gluon divergences in the resummed cross section that appear when $\alpha_{s}$ reaches the Landau pole. As we have discussed before, in a finite-order expansion, as presented in this paper, there are no divergences and the results are prescription independent.

There are three resummation prescriptions available in the literature. The earliest is the $x$-space formalism of Refs. [3, 31]. The resummation was performed at leading logarithmic (LL) accuracy in momentum space and a cutoff was chosen to avoid the divergence. In practice, the cutoff was chosen so that numerically the resummed result would agree with the expansion of the resummed cross section through NNLO. This approach was also used at NLL accuracy in Refs. [8, 9, 12]. The finite-order expansion is essentially the same whether the resummation is performed in momentum or moment space so, although the approach is quite different, in practice the numerical results from this approach are not inconsistent with the ones we are presenting here at LL and NLL accuracy.

The second prescription is principal value resummation, originally developed for Drell-Yan production in Ref. [32]. A principal value prescription is used to bypass the Landau pole. This approach was used at LL accuracy for top quark production in Refs. [1, 18]. Numerically the results are similar to those of Ref. [3]. This approach has not yet been used at NLL accuracy for top quark production at present.

The third approach is the minimal prescription of Ref. [5]. It has been applied at both LL [5] and NLL accuracy [10] to heavy quark production. Numerically it differs substantially

from the results of Refs. [3, 4]. As discussed in Refs. [5, 18] this difference emerges from extra subleading terms, which are kept in the minimal prescription approach, that come from the inversion of the resummed cross section from moment to momentum space.

Let us begin our study of subleading terms by rewriting the $\overline{\mathrm{MS}} \mathrm{NLO}$ corrections for $q \bar{q} \rightarrow$ $Q \bar{Q}$ in Eq. (3.1) in the shorthand notation

$$
\hat{\sigma}^{(1)}\left(s_{4}\right)=\sigma^{B} \frac{\alpha_{s}}{\pi}\left\{c_{1} \delta\left(s_{4}\right)+c_{2}\left[\frac{1}{s_{4}}\right]_{+}+c_{3}\left[\frac{\ln \left(s_{4} / m^{2}\right)}{s_{4}}\right]_{+}\right\},
$$

with $c_{3}=4 C_{F}, c_{2}=2 \operatorname{Re} \Gamma_{22}^{\prime q \bar{q}}-2 C_{F}+2 C_{F} \ln \left(s m^{2} /\left(t_{1} u_{1}\right)\right)-2 C_{F} \ln \left(\mu_{F}^{2} / m^{2}\right)$, and $c_{1}=c_{\overline{\mathrm{MS}}}^{(1) q \bar{q} \mathrm{~S}+\mathrm{V}}$. This result actually comes from the inversion of the moment space expression

$$
\hat{\sigma}^{(1)}(N)=\sigma^{B} \frac{\alpha_{s}}{\pi}\left\{c_{1}+c_{2} I_{0}(N)+c_{3} I_{1}(N)\right\}=\sigma^{B} \frac{\alpha_{s}}{\pi}\left\{c_{1}-c_{2} \ln \tilde{N}+\frac{c_{3}}{2}\left(\ln ^{2} \tilde{N}+\zeta_{2}\right)\right\}
$$

where $\tilde{N}=N e^{\gamma_{E}}$, with $\gamma_{E}$ the Euler constant, and $I_{0}$ and $I_{1}$ are given in Appendix A. Eq. (3.15) comes directly from the expansion of the resummed cross section in moment space in Eq. (2.7). 
Now, let us examine the NLO expansion at NLL accuracy, with $\mu_{F}=\mu_{R}=m$. At that accuracy, we keep only the $\ln ^{2} N$ and $\ln N$ terms in Eq. (3.15). Since $\ln N=\ln \tilde{N}-\gamma_{E}$, upon inversion to momentum space we get back the $\left[\ln \left(s_{4} / m^{2}\right) / s_{4}\right]_{+}$and $\left[1 / s_{4}\right]_{+}$terms in Eq. (3.14) plus the following extra terms:

$$
\sigma^{B} \frac{\alpha_{s}}{\pi}\left[-\frac{c_{3} \gamma_{E}^{2}}{2}+c_{2} \gamma_{E}-\frac{\zeta_{2}}{2} c_{3}\right] \delta\left(s_{4}\right) .
$$

But there are no terms involving $\gamma_{E}$ in the exact NLO calculation, i.e. in the term $c_{1}$ in Eq. (3.14) which comes from $\sigma_{\overline{\mathrm{MS}}}^{(1) q \overline{\mathrm{S}}+\mathrm{V}}$; therefore these terms are clearly unphysical. They should not appear in the cross section because of the definition of the $\overline{\mathrm{MS}}$ scheme. The $\gamma_{E}$ terms are an artifact of the inversion from moment to momentum space. Indeed, if we had kept NLL terms in $\ln \tilde{N}$ rather than $\ln N$, there would be no $\gamma_{E}$ terms. Also the coefficient of the $\zeta_{2}$ term is wrong. As can be seen from the full NLO corrections, it has the wrong sign. We can study the numerical effect of these extra terms on the cross section. At the Tevatron, with $\sqrt{S}=1.8 \mathrm{TeV}$ and $m=175 \mathrm{GeV} / \mathrm{c}^{2}$, the full NLO $S+V$ corrections, Eq. (3.1), are $1.26 \mathrm{pb}$ for $\mu_{F}=\mu_{R}=m$, see Table 1. At NLL accuracy, the corrections are $1.31 \mathrm{pb}$. If we include all the unphysical terms of Eq. (3.16), the NLL corrections become $0.39 \mathrm{pb}$, clearly very far from the true size of the full $S+V$ corrections. Keeping only the $\zeta_{2}$ term in Eq. (3.16) we find $0.74 \mathrm{pb}$, which is closer to but still well below the full $S+V$ corrections. If we keep only the $\zeta_{2}$ term but with the opposite sign, as in the full corrections, we find $1.88 \mathrm{pb}$. It is clear that keeping unphysical subleading terms in the cross section can produce very misleading results. Even if we discard the unphysical terms and keep only some of the physical subleading terms we can still make erroneous predictions, especially if the coefficients are wrong. One of the greatest achievements (or, from another viewpoint, tests) of the formalism of theshold resummation at NLL and higher accuracy is that it accurately reproduces the exact NLO cross section both analytically and numerically. In fact, one may argue that only because of this agreement is threshold resummation worthwhile. After all, if the corrections to be resummed are not dominant then the necessity for resummation is greatly diminished. That holds not only for heavy quark production but also for other QCD processes such as direct photon [22], $\mathrm{W}+$ jet [20], and single-jet production [24]. Therefore it is important to ensure that we don't introduce terms, unphysical or otherwise, that would spoil this agreement.

We can extend our study of subleading logarithms to NNLO. The NNLO threshold corrections in moment space are given in shorthand notation by

$$
\begin{aligned}
\hat{\sigma}^{(2)}(N)= & \sigma^{B} \frac{\alpha_{s}^{2}}{2 \pi^{2}}\left\{\frac{c_{3}^{2}}{4} \ln ^{4} \tilde{N}-c_{3} c_{2} \ln ^{3} \tilde{N}+\left[c_{3}\left(c_{1}+\frac{1}{2} \zeta_{2} c_{3}\right)+c_{2}^{2}\right] \ln ^{2} \tilde{N}\right. \\
& \left.-2 c_{2} \ln \tilde{N}\left(c_{1}+\frac{1}{2} \zeta_{2} c_{3}\right)+\left(c_{1}+\frac{1}{2} \zeta_{2} c_{3}\right)^{2}+\tilde{F}\left(\beta_{0}, \Gamma_{S}^{2}, K, 2-\text { loop }\right)\right\}
\end{aligned}
$$

i.e. by the square of the terms in curly brackets in Eq. (3.15), plus a function $\tilde{F}$ that comprises the $\beta_{0}$ terms that come from changing the argument in the running coupling, $\alpha_{s}\left(\mu^{\prime 2}\right)=$ $\alpha_{s}\left(\mu^{2}\right)\left[1-\beta_{0} \ln \left(\mu^{\prime 2} / \mu^{2}\right)\left(\alpha_{s}\left(\mu^{2}\right) /(4 \pi)\right]\right.$; the two-loop $K$ terms, with $K$ defined below Eq. (2.8); square terms from the off-diagonal soft anomalous dimension matrix elements; and two-loop $\Gamma_{S}$ and other terms (we have also absorbed in $\tilde{F}$ a term $-T_{1}^{2}$, with $T_{1}=c_{1}(\mu=m)$ ). Note that apart from the $K$ terms, the other two-loop terms appear only beyond NNLL accuracy 
and are not known at present. We then rewrite Eq. 3.17) in terms of $I_{3}, I_{2}, I_{1}$, and $I_{0}$ defined in Appendix A.

We can then immediately invert back to momentum space and find

$$
\begin{aligned}
\hat{\sigma}^{(2)}\left(s_{4}\right)= & \sigma^{B} \frac{\alpha_{s}^{2}}{\pi^{2}}\left\{\frac{1}{2} c_{3}^{2}\left[\frac{\ln ^{3}\left(s_{4} / m^{2}\right)}{s_{4}}\right]_{+}+\frac{3}{2} c_{3} c_{2}\left[\frac{\ln ^{2}\left(s_{4} / m^{2}\right)}{s_{4}}\right]_{+}\right. \\
& +\left(c_{3} c_{1}+c_{2}^{2}-\zeta_{2} c_{3}^{2}\right)\left[\frac{\ln \left(s_{4} / m^{2}\right)}{s_{4}}\right]_{+}+\left(c_{2} c_{1}-\zeta_{2} c_{2} c_{3}+\zeta_{3} c_{3}^{2}\right)\left[\frac{1}{s_{4}}\right]_{+} \\
& \left.+\left(\frac{c_{1}^{2}}{2}-\frac{c_{2}^{2}}{2} \zeta_{2}+\frac{1}{4} c_{3}^{2} \zeta_{2}^{2}+\zeta_{3} c_{3} c_{2}-\frac{3}{4} \zeta_{4} c_{3}^{2}\right) \delta\left(s_{4}\right)+F\left(\beta_{0}, \Gamma_{S}^{2}, K, 2-\text { loop }\right)\right\} .
\end{aligned}
$$

Here $F$ denotes the terms that come from the inversion of $\tilde{F}$ and starts contributing at NLL and higher accuracy. One can easily see that this form, with the appropriate explicit expression for $F$, agrees with the NNLO-NNLL expansion given in Eq. (3.8), including the additional scale terms of Eq. (3.11). Clearly we don't know all the $\left[1 / s_{4}\right]_{+}$and $\delta\left(s_{4}\right)$ terms because of unknown two-loop corrections in $F$. Of course there are no $\gamma_{E}$ terms in the NNLO cross section. This is also known from the two-loop Drell-Yan cross section [33, 34, 35]: many of the NNLO terms are the same as for top quark production since the exponent in the resummed cross section that comes from the incoming partons is universal; and there are no $\gamma_{E}$ terms. Again, this follows from the definition of the $\overline{\mathrm{MS}}$ scheme. These terms are clearly unphysical at both NLO and NNLO and indeed at any higher order.

Now, let us see what happens if one keeps the logarithms only at a certain accuracy. At NNLL accuracy, we keep the $\ln ^{4} N, \ln ^{3} N$, and $\ln ^{2} N$ terms in $\hat{\sigma}^{(2)}(N)$, Eq. (3.17). Then, the subleading terms from the inversion to momentum space are:

$$
\begin{aligned}
& \sigma^{B} \frac{\alpha_{s}^{2}}{\pi^{2}}\left\{\left(\zeta_{3} c_{3}^{2}-\frac{3}{2} c_{3} c_{2} \zeta_{2}\right)\left[\frac{1}{s_{4}}\right]_{+}\right. \\
& \left.+\delta\left(s_{4}\right)\left(\frac{c_{3}^{2} \zeta_{2}^{2}}{8}+c_{3} c_{2} \zeta_{3}-\frac{3}{4} c_{3}^{2} \zeta_{4}-\frac{\zeta_{2}}{2} c_{2}^{2}-\frac{\zeta_{2}}{2} c_{3} c_{1}-2 \zeta_{2} \Gamma_{12}^{\prime q \bar{q}} \Gamma_{21}^{\prime q \bar{q}}\right)\right\}
\end{aligned}
$$

plus

$$
\begin{aligned}
& \sigma^{B} \frac{\alpha_{s}^{2}}{\pi^{2}}\left\{\beta_{0} C_{F} \zeta_{2}\left[\frac{1}{s_{4}}\right]_{+}\right. \\
& \left.+\delta\left(s_{4}\right)\left[-\frac{2}{3} \beta_{0} C_{F} \zeta_{3}-\zeta_{2}\left(-\frac{\beta_{0}}{4} c_{2}+C_{F} K\right)\right]\right\}
\end{aligned}
$$

plus

$$
\begin{aligned}
& \sigma^{B} \frac{\alpha_{s}^{2}}{\pi^{2}}\left\{\gamma_{E}\left(\beta_{0} C_{F} \gamma_{E}+4 \Gamma_{12}^{\prime q \bar{q}} \Gamma_{21}^{\prime q \bar{q}}-\frac{\beta_{0}}{2} c_{2}+2 C_{F} K\right)\left[\frac{1}{s_{4}}\right]_{+}\right. \\
& \left.+\delta\left(s_{4}\right) \gamma_{E}^{2}\left(\frac{2}{3} \beta_{0} C_{F} \gamma_{E}+2 \Gamma_{12}^{\prime q \bar{q}} \Gamma_{21}^{\prime q \bar{q}}-\frac{\beta_{0}}{4} c_{2}+C_{F} K\right)\right\} \\
& +\sigma^{B} \frac{\alpha_{s}^{2}}{\pi^{2}}\left\{\left[\frac{c_{3}^{2}}{2} \gamma_{E}^{3}-\frac{3}{2} c_{3} c_{2} \gamma_{E}^{2}+2 \gamma_{E}\left(c_{3} c_{1}+c_{3}^{2} \frac{\zeta_{2}}{2}+c_{2}^{2}\right)\right]\left[\frac{1}{s_{4}}\right]_{+}\right. \\
& \left.+\delta\left(s_{4}\right)\left[\frac{3}{8} c_{3}^{2} \gamma_{E}^{4}-c_{3} c_{2} \gamma_{E}^{3}+\gamma_{E}^{2}\left(c_{3} c_{1}+c_{3}^{2} \frac{\zeta_{2}}{2}+c_{2}^{2}\right)\right]\right\} .
\end{aligned}
$$


The subleading $\zeta$ terms in Eq. (3.19) appear also in the full cross section of Eq. (3.18). However, a comparison between these two equations shows that some of these terms have the wrong coefficients. The terms in Eq. (3.21) are again the unphysical $\gamma_{E}$ terms that should not appear at any order of the perturbative series. Again, if we had kept NNLL terms in $\ln \tilde{N}$ rather than $\ln N$, there would be no $\gamma_{E}$ terms. The $\beta_{0}$ and $K$ terms in Eq. (3.20) would also be absent in an exact calculation. This is because they appear in integrals of the form of Eq. (A.1), which upon inversion to momentum space should give back the original "plus" distributions with no subleading terms.

We can again study the numerical effect of these extra subleading terms. In the following, we keep $\mu_{F}=\mu_{R}=m$. At the Tevatron, with $\sqrt{S}=1.8 \mathrm{TeV}$ and $m=175 \mathrm{GeV} / \mathrm{c}^{2}$, the $\overline{\mathrm{MS}}$ NNLO-NNLL corrections for $q \bar{q} \rightarrow t \bar{t}$ are $0.80 \mathrm{pb}$ as we saw in Table 1 . If we keep also the subleading $\zeta$ terms in Eq. (3.19) the result becomes $0.39 \mathrm{pb}$. If we keep the subleading terms in both Eqs. (3.19) and (3.20), the corrections become $0.13 \mathrm{pb}$. Finally, if we include all subleading terms, Eqs. (3.19), (3.20), and (3.21) the corrections become $0.08 \mathrm{pb}$. This last result is similar to the result presented in Ref. [10] (note that different parton densities are used; also our formalism resums the fully differential cross section while [10 resums only the total cross section; the latter approach introduces some additional errors, see the discussion in Ref. [36]). Clearly the inclusion of the unphysical $\gamma_{E}$ and other terms decreases the NNLO-NNLL corrections by a factor of ten. The effects of these unphysical terms are much bigger than those of the LL, NLL, and NNLL terms. It is difficult to accept a result in which unphysical subleading terms dominate the three leading powers of the logarithms. Such a result defies the meaning of leading level, next-to-leading level and so on. And as was evidenced by the NLO exercise, a result with these subleading terms substantially underestimates the correct value for the cross section. We also note that if we keep the subleading $\zeta$ terms from the inversion at full accuracy, as in Eq. (3.18), the corrections are $0.45 \mathrm{pb}$, much closer to the NNLO-NNLL result. Of course, we can't derive the full NNLO cross section beyond NNLL accuracy because of missing two-loop terms, but this certainly indicates that the corrections with subleading terms tend to get larger the better the accuracy.

We can also repeat this exercise at NLL accuracy. Here we will disregard the $\gamma_{E}$ and $\beta_{0}, K$ terms. At NLL accuracy for $\hat{\sigma}^{(2)}$, the subleading $\zeta$ terms from the inversion are

$$
\begin{aligned}
& \sigma^{B} \frac{\alpha_{s}^{2}}{\pi^{2}}\left\{-\frac{3}{2} \zeta_{2} c_{3}^{2}\left[\frac{\ln \left(s_{4} / m^{2}\right)}{s_{4}}\right]_{+}+\left(\zeta_{3} c_{3}^{2}-\frac{3}{2} c_{3} c_{2} \zeta_{2}\right)\left[\frac{1}{s_{4}}\right]_{+}\right. \\
& \left.+\delta\left(s_{4}\right)\left[\frac{3}{8} c_{3}^{2}\left(\zeta_{2}^{2}-2 \zeta_{4}\right)+c_{3} c_{2} \zeta_{3}\right]\right\} .
\end{aligned}
$$

Again, the subleading terms above appear also in the full cross section of Eq. (3.18) but some of these terms have the wrong coefficients. As we saw in Table 1, the NNLO-NLL corrections are $1.01 \mathrm{pb}$. If one adds the subleading terms above, the result becomes $0.23 \mathrm{pb}$. Our conclusions remain the same. We also note that if we keep only the $\left[\left(\ln \left(s_{4} / m^{2}\right)\right) / s_{4}\right]_{+}$subleading terms from the inversion, we find that the corrections are $0.58 \mathrm{pb}$, quite different from the result we get $(0.80 \mathrm{pb})$ when we calculate the full NNLL terms (if we also include the unphysical terms the disagreement is far worse). In addition, we observe that if we perform a LL calculation and then include subleading NLL (i.e. $\left.\left[\left(\ln ^{2}\left(s_{4} / m^{2}\right)\right) / s_{4}\right]_{+}\right)$terms from the inversion involving $\gamma_{E}$, the corrections become $0.28 \mathrm{pb}$ versus the $1.01 \mathrm{pb}$ that we find with a full NLL calculation. All these exercises highlight the numerical problems that are encountered if one includes unjustified subleading terms in the expansion. 
As is noted in Ref. [37], the integrals of the leading logarithmic distributions with any smooth function, such as the convolution with parton distributions, produce factorial contributions at $n$th order of the form $\alpha_{s}^{n}(2 n-1) ! / n !+\ldots$. These factorial terms naturally arise in both the exact cross section at any fixed order and in the finite-order expansions of the resummed cross section. In the minimal prescription of Ref. [5] subleading terms are kept in the resummed cross section and its expansion in order to avoid certain power corrections, arising from these factorial contributions, which have been shown to be absent in the Drell Yan cross section in Refs. [38, 39]. This is indeed a problem that deserves attention as pointed out in Ref. [5]. However, the absence of unphysical power corrections does not require the introduction in the expansion of unphysical terms, which, as we have seen above, may greatly underestimate the true value of the cross section. The exact cross section does not have these power corrections but also it does not have the $\gamma_{E}$ terms, only the $\zeta$ terms, as we have seen explicitly at NLO and NNLO. Moreover, one has to be careful not to introduce extra terms, even physical ones, that produce erroneous numerical results. At low orders, with $n=1,2$ the factorial contributions are negligible or small anyway, and certainly smaller than other terms in the expansion (see also discussion in Ref. [18]). At higher orders of course the factorial contribution grows and moreover we have ever increasing numbers of uknown coefficients of subleading logarithms (which actually may be more important numerically than the factorial terms). Therefore we stop the expansion at NNLO, thus avoiding the theoretical problem with power corrections. At NLO and NNLO we can trust the perturbative expansion, as we have determined all logarithmic coefficients. Our numerical analyses above confirm that. We also note that in our approach the results for the NNLO corrections do not change substantially when going from LL to NNLL accuracy (they change from $0.59 \mathrm{pb}$ to $0.80 \mathrm{pb}$ ) while the corresponding results with all subleading terms change by almost an order of magnitude (that can also be seen by comparing Table 1 of Ref. [5] with Table 2 of Ref. [10]). The relative stability of our results versus logarithmic accuracy is an additional justification of our approach. We can investigate keeping the $\zeta$ terms as a rough estimate of error, as we have done above, but we should keep in mind that they may not necessarily offer an improvement on the calculation as evidenced from the NLO results. Therefore, we find it best in the numerical analyses presented in this paper not to retain any terms beyond NNLL accuracy. Thus, we do not find the very fast convergence of the higher order corrections that is claimed in Refs. [5, 10].

In a recent paper [40] it is argued that threshold enhancements are dominated by the region where the hierarchy among different powers of the threshold logarithms is lost, and therefore NLL resummation is not reliable. Our numerical results at fixed order do not agree with this claim, although it is certainly true that the coefficients of lower powers of the logarithm can be large. Moreover, we note that even if the hierarchy among different powers of the logarithms were lost, at NNLO we have determined the coefficients of all the powers of the logarithms, so our results are reliable regardless. Beyond NNLO, however, there are subleading powers of logarithms with undetermined coefficients, which can be large, and then the ambiguities with regard to the effect of subleading terms increase. Therefore, as discussed above, for detailed numerical results we prefer to stop the expansion at NNLO. 


\subsection{NNLO-NNLL threshold corrections for $g g \rightarrow Q \bar{Q}$}

For the $g g$ channel in the $\overline{\mathrm{MS}}$ scheme the NNLO-NNLL corrections are

$$
\begin{aligned}
& \hat{\sigma}_{g g \rightarrow Q \bar{Q}}^{\overline{\mathrm{MS}}(2)}\left(s_{4}, m^{2}, s, t_{1}, u_{1}, \mu_{F}, \mu_{R}\right)=\sigma_{g g \rightarrow Q \bar{Q}}^{B}\left(\frac{\alpha_{s}\left(\mu_{R}^{2}\right)}{\pi}\right)^{2}\left\{8 C_{A}^{2}\left[\frac{\ln ^{3}\left(s_{4} / m^{2}\right)}{s_{4}}\right]_{+}\right. \\
& \left.+\left[-\beta_{0} C_{A}-12 C_{A}^{2} \ln \left(\frac{\mu_{F}^{2}}{m^{2}}\right)\right]\left[\frac{\ln ^{2}\left(s_{4} / m^{2}\right)}{s_{4}}\right]_{+}\right\} \\
& +\frac{\alpha_{s}^{4}\left(\mu_{R}^{2}\right)}{\pi} K_{g g} B_{\mathrm{QED}}\left[\frac{\ln ^{2}\left(s_{4} / m^{2}\right)}{m^{2}}\right]_{+} C_{A} 3\left(N_{c}^{2}-1\right)\left\{\frac{\left(t_{1}^{2}+u_{1}^{2}\right)}{s^{2}}\right. \\
& \times\left[N_{c}^{2} \ln \left(\frac{m^{2} s}{t_{1} u_{1}}\right)-2 N_{c}\left(C_{F}-\frac{C_{A}}{2}\right) \operatorname{Re} L_{\beta}-2 N_{c} C_{F}\right]+2 \frac{C_{F}}{N_{c}} \\
& \left.+2 \frac{1}{N_{c}}\left(C_{F}-C_{A}\right) \operatorname{Re} L_{\beta}+N_{c}^{2} \frac{\left(t_{1}^{2}-u_{1}^{2}\right)}{s^{2}} \ln \left(\frac{u_{1}}{t_{1}}\right)\right\} \\
& +\left[\frac{\ln \left(s_{4} / m^{2}\right)}{m^{2}}\right]_{+}\left\{( \frac { \alpha _ { s } ( \mu _ { R } ^ { 2 } ) } { \pi } ) ^ { 2 } \sigma _ { g g \rightarrow Q \overline { Q } } ^ { B } \left[\beta_{0}\left(C_{A}+C_{A} \ln \left(\frac{\mu_{R}^{2}}{m^{2}}\right)+C_{A} \ln \left(\frac{t_{1} u_{1}}{m^{2} s}\right)\right)\right.\right. \\
& \left.+2 C_{A} K-16 \zeta_{2} C_{A}^{2}+4 C_{A} c_{\overline{\mathrm{MS}}}^{(1) g \mathrm{~S}+\mathrm{V}}\right] \\
& +\frac{\alpha_{s}^{4}\left(\mu_{R}^{2}\right)}{2 \pi\left(N_{c}^{2}-1\right)} B_{\mathrm{QED}}\left(1-\frac{2 t_{1} u_{1}}{s^{2}}\right)\left[4 N_{c}\left(\operatorname{Re}_{22}^{\prime g g}-C_{A}-C_{A} \ln \left(\frac{\mu_{F}^{2}}{s}\right)-C_{A} \ln \left(\frac{t_{1} u_{1}}{m^{4}}\right)\right)^{2}\right. \\
& \left.+\frac{N_{c}}{4}\left(N_{c}^{2}+4\right)\left(\Gamma_{31}^{\prime g g}\right)^{2}-\beta_{0} N_{c} \operatorname{Re} \Gamma_{22}^{\prime g g}\right] \\
& +\frac{\alpha_{s}^{4}\left(\mu_{R}^{2}\right)}{2 \pi\left(N_{c}^{2}-1\right)} B_{\mathrm{QED}}\left(\frac{t_{1}^{2}-u_{1}^{2}}{s^{2}}\right)\left[4 \Gamma_{31}^{g g}\left(\operatorname{Re}_{11}^{\prime g g}-C_{A}-C_{A} \ln \left(\frac{\mu_{F}^{2}}{s}\right)-C_{A} \ln \left(\frac{t_{1} u_{1}}{m^{4}}\right)\right)\right. \\
& \left.+2\left(N_{c}^{2}-2\right) \Gamma_{31}^{\prime g g}\left(\operatorname{Re}_{22}^{\prime g g}-C_{A}-C_{A} \ln \left(\frac{\mu_{F}^{2}}{s}\right)-C_{A} \ln \left(\frac{t_{1} u_{1}}{m^{4}}\right)\right)-\beta_{0} \frac{N_{c}^{2}}{4} \Gamma_{31}^{\prime g g}\right] \\
& +\frac{\alpha_{s}^{4}\left(\mu_{R}^{2}\right)}{2 \pi\left(N_{c}^{2}-1\right)} B_{\mathrm{QED}}\left[\frac{4}{N_{c}}\left(\operatorname{Re}_{11}^{\prime g g}-C_{A}-C_{A} \ln \left(\frac{\mu_{F}^{2}}{s}\right)-C_{A} \ln \left(\frac{t_{1} u_{1}}{m^{4}}\right)\right)^{2}-\frac{N_{c}}{2}\left(\Gamma_{31}^{\prime g g}\right)^{2}\right. \\
& \left.\left.-\frac{8}{N_{c}}\left(\operatorname{Re}^{\prime g}{ }_{22}^{g g}-C_{A}-C_{A} \ln \left(\frac{\mu_{F}^{2}}{s}\right)-C_{A} \ln \left(\frac{t_{1} u_{1}}{m^{4}}\right)\right)^{2}-\beta_{0} \frac{1}{N_{c}} \operatorname{Re}\left(\Gamma_{11}^{\prime g g}-2 \Gamma_{22}^{\prime g g}\right)\right]\right\} \\
& +\mathcal{O}\left(\left[\frac{1}{s_{4}}\right]_{+}\right)
\end{aligned}
$$

where $c \frac{(1) g g \mathrm{~S}+\mathrm{V}}{\mathrm{MS}}$ is defined in analogy to Eq. (3.9), and the elements of the soft anomalous dimension matrix (dropping gauge-dependent terms and an overall $\alpha_{s} / \pi$ ) are

$$
\begin{aligned}
\operatorname{Re}_{11}^{\prime g g} & =-C_{F}\left(\operatorname{Re} L_{\beta}+1\right)+C_{A}, \quad \Gamma_{31}^{\prime g g}=\ln \left(\frac{u_{1}^{2}}{t_{1}^{2}}\right), \\
\operatorname{Re}_{22}^{\prime g g} & =-C_{F}\left(\operatorname{Re} L_{\beta}+1\right)+\frac{C_{A}}{2}\left[2+\ln \left(\frac{t_{1} u_{1}}{m^{2} s}\right)+\operatorname{Re} L_{\beta}\right] .
\end{aligned}
$$


As for the $q \bar{q}$ channel, we can also derive at NNLL accuracy additional $\left[1 / s_{4}\right]_{+}$and $\delta\left(s_{4}\right)$ terms involving the scale. These terms are

$$
\begin{aligned}
\sigma_{g g \rightarrow Q \bar{Q}}^{B}\left(\frac{\alpha_{s}\left(\mu_{R}^{2}\right)}{\pi}\right)^{2}\left[\frac{1}{s_{4}}\right]_{+}\left\{\ln ^{2}\left(\frac{\mu_{F}^{2}}{m^{2}}\right) C_{A}\left[\frac{5}{4} \beta_{0}-2 C_{A} \ln \left(\frac{t_{1} u_{1}}{m^{4}}\right)\right]-\frac{3}{2} C_{A} \beta_{0} \ln \left(\frac{\mu_{R}^{2}}{m^{2}}\right) \ln \left(\frac{\mu_{F}^{2}}{m^{2}}\right)\right. \\
+\ln \left(\frac{\mu_{F}^{2}}{m^{2}}\right)\left[-2 C_{A} \hat{T}_{\overline{\mathrm{MS}}}^{(1) g g}+8 C_{A}^{2} \zeta_{2}-C_{A} K+\beta_{0} N_{c}+\beta_{0} N_{c} \ln \left(\frac{t_{1} u_{1}}{m^{2} s}\right)\right. \\
\left.\quad-2 N_{c}^{2} \ln ^{2}\left(\frac{t_{1} u_{1}}{m^{4}}\right)-2 N_{c}^{2} \ln \left(\frac{t_{1} u_{1}}{m^{4}}\right) \ln \left(\frac{m^{2}}{s}\right)-2 N_{c}^{2} \ln \left(\frac{t_{1} u_{1}}{m^{4}}\right)\right] \\
\left.+\frac{3}{2} C_{A} \beta_{0} \ln \left(\frac{\mu_{R}^{2}}{m^{2}}\right)\left[\ln \left(\frac{t_{1} u_{1}}{m^{2} s}\right)+1\right]\right\} \\
+\frac{\alpha_{s}^{4}\left(\mu_{R}^{2}\right)}{\pi} \frac{B_{\mathrm{QED}}}{N_{c}\left(N_{c}^{2}-1\right)}\left[\frac{1}{s_{4}}\right]+\left[\operatorname{Re}^{\prime g g}-2 \operatorname{Re} \Gamma_{22}^{g g}+\left(1-\frac{2 t_{1} u_{1}}{s^{2}}\right) N_{c}^{2} \operatorname{Re}_{22}^{\prime g g}+\frac{N_{c}^{3}}{4} \Gamma_{31}^{\prime g g} \frac{\left(t_{1}^{2}-u_{1}^{2}\right)}{s^{2}}\right] \\
\times\left[C_{A} \ln \left(\frac{t_{1} u_{1}}{m^{4}}\right) \ln \left(\frac{\mu_{F}^{2}}{m^{2}}\right)+\frac{\beta_{0}}{2} \ln \left(\frac{\mu_{R}^{2}}{\mu_{F}^{2}}\right)+\frac{\beta_{0}}{4} \ln \left(\frac{\mu_{R}^{2}}{m^{2}}\right)\right] \\
+\sigma_{g g \rightarrow Q \bar{Q}}^{B}\left(\frac{\alpha_{s}\left(\mu_{R}^{2}\right)}{\pi}\right)^{2} \delta\left(s_{4}\right)\left[\left(-2 \zeta_{2} C_{A}^{2}+\frac{1}{2} C_{A}^{2} \ln ^{2}\left(\frac{t_{1} u_{1}}{m^{4}}\right)-\frac{5}{8} \beta_{0} C_{A} \ln \left(\frac{t_{1} u_{1}}{m^{4}}\right)\right) \ln ^{2}\left(\frac{\mu_{F}^{2}}{m^{2}}\right)\right. \\
\left.\quad+\frac{3}{4} \beta_{0} C_{A} \ln \left(\frac{t_{1} u_{1}}{m^{4}}\right) \ln \left(\frac{\mu_{F}^{2}}{m^{2}}\right) \ln \left(\frac{\mu_{R}^{2}}{m^{2}}\right)+\frac{3}{16} \beta_{0}^{2} \ln ^{2}\left(\frac{\mu_{R}^{2}}{\mu_{F}^{2}}\right)\right],
\end{aligned}
$$

where $\hat{T}_{\overline{\mathrm{MS}}}^{(1) g g}$ is defined in analogy to its counterpart in the $q \bar{q}$ channel.

At the Tevatron, with $\sqrt{S}=1.8 \mathrm{TeV}$ and $\mu_{F}=\mu_{R}=m=175 \mathrm{GeV} / \mathrm{c}^{2}$, the exact NLO cross section for the $g g$ channel is $0.55 \mathrm{pb}$ and the NNLO-NNLL corrections provide an additional $0.32 \mathrm{pb}$. The relative size of the NNLO corrections for the $g g$ channel compared to NLO is much greater than for the $q \bar{q}$ channel. This is because of the different color coefficients in the expressions for the two channels, as is obvious from the coefficients of the leading logarithms.

NNLO results for the $g g$ channel in PIM kinematics are presented in Appendix C (see also Ref. [13]).

\subsection{Top production at the Tevatron}

In this subsection we add the numerical contributions from the $q \bar{q}$ and $g g$ partonic channels and present some numerical results for the top quark total cross section and transverse momentum distributions at the Tevatron (see Appendix B for a discussion of the hadronic calculation). We use the CTEQ5M parton densities [30].

In Fig. 3 we plot the Born term and the NLO and NNLO corrections for top quark production at the Tevatron with $\sqrt{S}=1.8 \mathrm{TeV}$ as a function of the top mass. At NLO we show the exact corrections as well as the NLL threshold corrections and the full $\mathrm{S}+\mathrm{V}$ threshold corrections. At NNLO we show results with both NLL and NNLL accuracy.

In Fig. 4 we plot the exact NLO and the NNLO-NNLL $\overline{\mathrm{MS}}$ top quark cross section at the Tevatron with $\sqrt{S}=1.8 \mathrm{TeV}$ as a function of the top mass. We note a dramatic decrease of the scale dependence of the cross section when we include the NNLO-NNLL corrections. We observe that the NNLO cross section is uniformly above the NLO cross section for all scale choices (we stress that there is no field-theoretical reason for the NNLO results to lie within the 


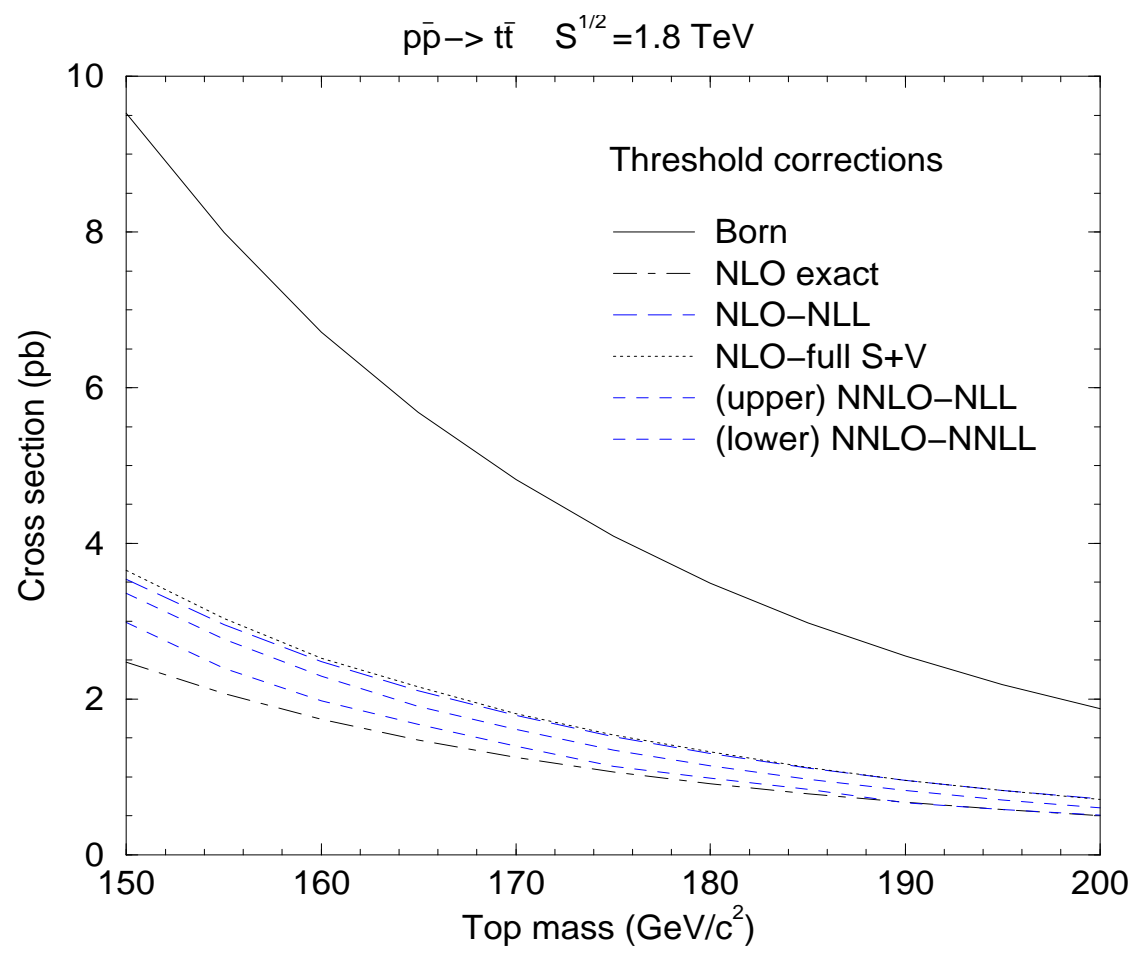

Fig. 3. The Born, NLO, and NNLO corrections for top quark production at the Tevatron with $\sqrt{S}=1.8 \mathrm{TeV}$.

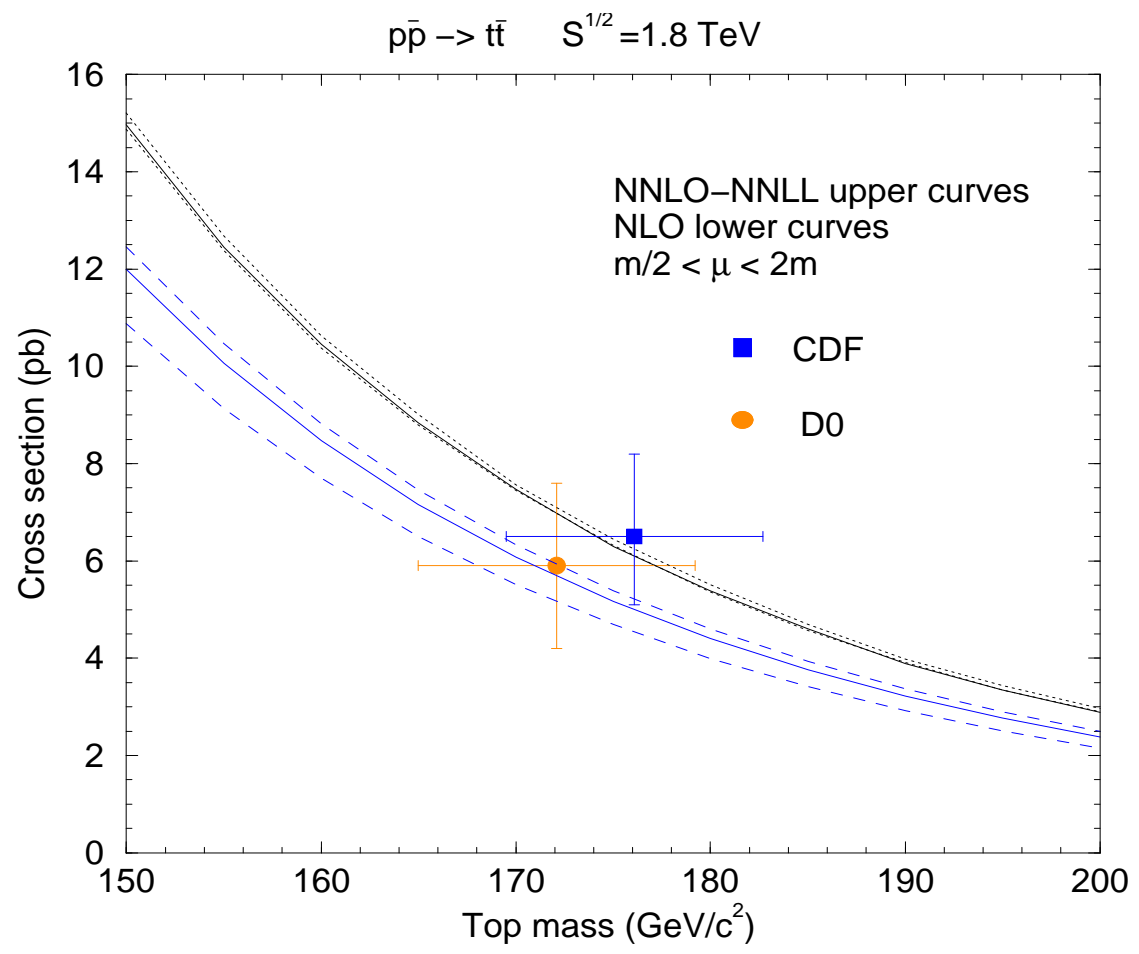

Fig. 4. The total cross section for top quark production at the Tevatron with $\sqrt{S}=1.8 \mathrm{TeV}$. The labels are as in Fig. 1. Recent results from CDF and D0 are also shown. 


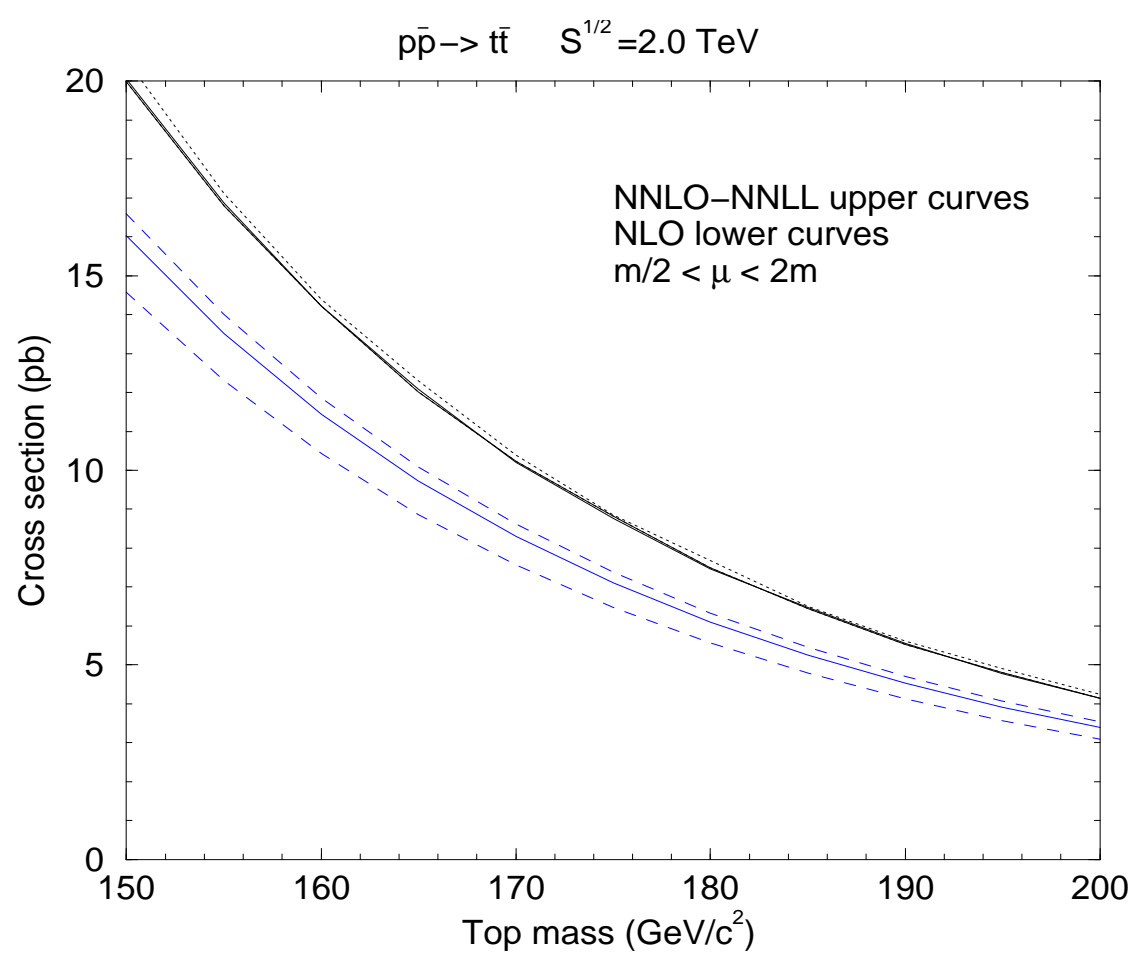

Fig. 5. The total cross section for top quark production at the Tevatron with $\sqrt{S}=2.0 \mathrm{TeV}$. The labels are as in Fig. 1.

NLO results). We also show recent results from CDF [41] and D0 442 and note the agreement between experiment and theory. In Fig. 5 we show the corresponding results for the upgraded Tevatron with $\sqrt{S}=2.0 \mathrm{TeV}$.

In Table 2 we list the exact NLO and the NNLO-NNLL total cross sections in pb for top quark production at the Tevatron with $\sqrt{S}=1.8 \mathrm{TeV}$ and $2.0 \mathrm{TeV}$, a top mass $m=175$ $\mathrm{GeV} / c^{2}$, and scale $\mu=m, m / 2,2 m$. The NNLO-NNLL cross section with $\sqrt{S}=1.8 \mathrm{TeV}$ is $6.3 \mathrm{pb}$ versus $5.2 \mathrm{pb}$ at NLO, an enhancement of over $20 \%$, at $\mu=m$. Good agreement is observed with recent results from CDF: $\sigma=6.5_{-1.4}^{+1.7}$ pb with $m=176.1 \pm 6.6 \mathrm{GeV} / c^{2}$ [41; and D0: $\sigma=5.9 \pm 1.7 \mathrm{pb}$ with $m=172.1 \pm 7.1 \mathrm{GeV} / c^{2}$ 42. Similar enhancements are noted for the upgraded Tevatron energy.

\begin{tabular}{|c|rl|rc|}
\hline$p \bar{p} \rightarrow t \bar{t}$ & \multicolumn{2}{|c|}{$\sqrt{S}=1.8 \mathrm{TeV}$} & \multicolumn{2}{c|}{$\sqrt{S}=2.0 \mathrm{TeV}$} \\
\hline$\mu=\mu_{F}=\mu_{R}$ & NLO & NNLO & NLO & NNLO \\
\hline$\mu=m / 2$ & 5.4 & 6.4 & 7.4 & 8.9 \\
\hline$\mu=m$ & 5.2 & 6.3 & 7.1 & 8.8 \\
\hline$\mu=2 m$ & 4.7 & 6.3 & 6.5 & 8.8 \\
\hline
\end{tabular}

Table 2: The hadronic $t \bar{t}$ production cross section in pb for $p \bar{p}$ collisions with $\sqrt{S}=1.8 \mathrm{TeV}$ and $2.0 \mathrm{TeV}$, and $m=175 \mathrm{GeV} / c^{2}$.

We would like to stress that the significantly reduced scale dependence should not be interpreted as an equivalent reduction of the uncertainty in the value of the cross section. Other sources of error such as from parton distributions, subleading logarithms, and distance from 

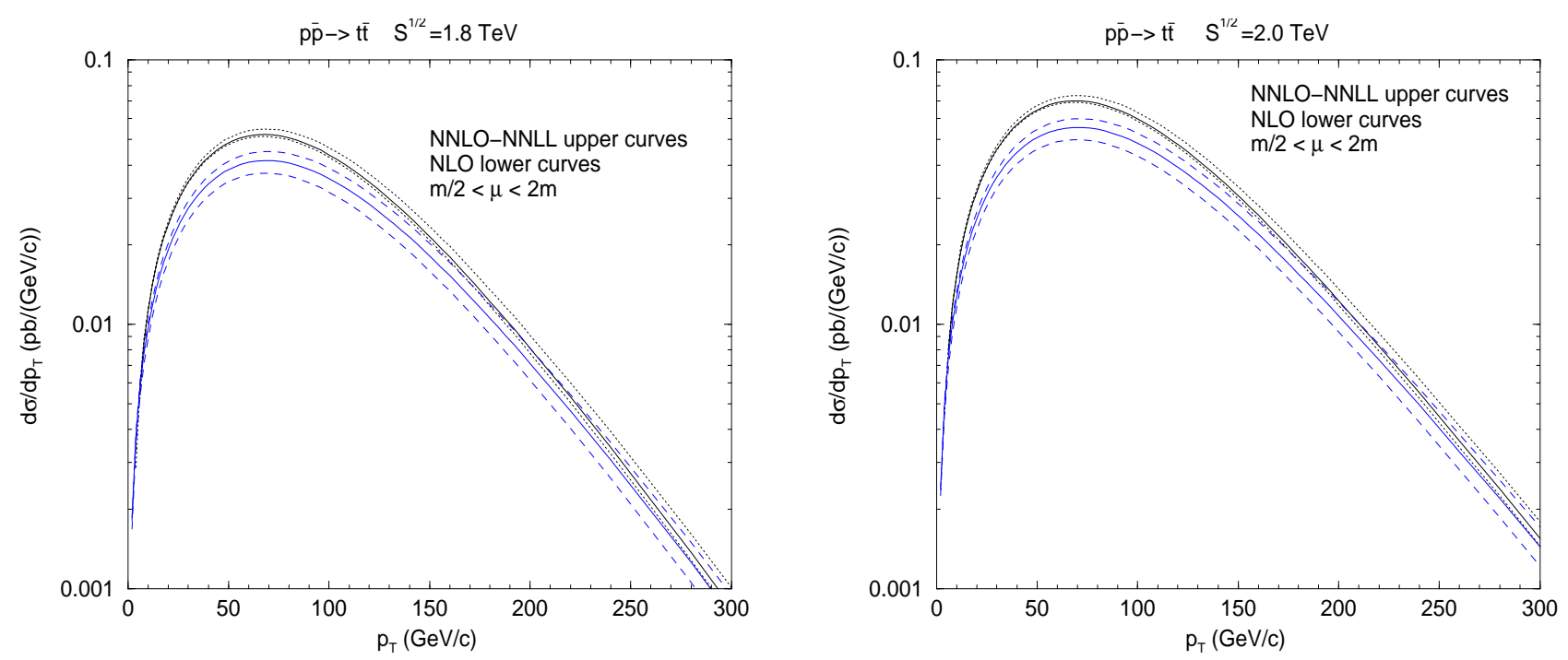

Fig. 6. Top quark transverse momentum distribution at the Tevatron, with $\sqrt{S}=1.8 \mathrm{TeV}$ and 2.0 TeV, and $m=175 \mathrm{GeV} / c^{2}$. The labels are as in Fig. 1.

threshold, can provide more uncertainty than the scale variation; and those errors cannot all be calculated precisely at present. This is why we do not give more than one significant figure after the decimal point in the numbers of Table 2. However, it is gratifying to see that perturbation theory behaves as we would expect it to [43]: at higher orders the scale variation decreases. Since the effect of subleading logarithms is the greatest uncertainty in the calculation, the total $t \bar{t}$ cross section can be written with a minimal error estimate as $6.3_{-0.4}^{+0.1} \mathrm{pb}$ at $\sqrt{S}=1.8 \mathrm{TeV}$ and as $8.8_{-0.5}^{+0.1} \mathrm{pb}$ at $\sqrt{S}=2.0 \mathrm{TeV}$, where the larger lower error indicates the uncertainty from subleading terms.

Our formalism allows the calculation of any relevant differential cross section. Transverse momentum and rapidity distributions with leading logarithmic resummation have been presented for top production at the Tevatron in Ref. [44]. The exact NLO and the NNLO-NNLL top quark transverse momentum $\left(p_{T}=t_{1} u_{1} / s^{2}-m^{2}\right)$ distributions at the Tevatron, with $\sqrt{S}=1.8 \mathrm{TeV}$ and $2.0 \mathrm{TeV}$, and $m=175 \mathrm{GeV} / c^{2}$ are shown in Fig. 6, again in the $\overline{\mathrm{MS}}$ scheme. We note an overall enhancement at NNLO with little change of shape. Similar conclusions are also reached for the rapidity distributions [45].

Finally, we note that threshold resummation is also relevant for bottom quark production at the HERA-B experiment. Leading logarithmic resummed results for the bottom quark total cross section and differential distributions have been presented in Refs. [27, 46]; for the NLL resummed cross section see Refs. [8, 10]. At NNLO-NNLL with $\sqrt{S}=41.6 \mathrm{GeV}$ and $\mu=m=4.75 \mathrm{GeV} / c^{2}$ we find a cross section for that experiment of $42 \mathrm{nb}$, while the NLO cross section is $18 \mathrm{nb}$.

\section{$4 \quad \mathrm{~N}^{3} \mathrm{LO}$ threshold corrections}

We now go beyond the NNLO corrections and expand the resummed cross section to next-tonext-to-next-to-leading order at NNLL accuracy. 
For the $q \bar{q}$ channel in the $\overline{\mathrm{MS}}$ scheme the $\mathrm{N}^{3} \mathrm{LO}-\mathrm{NNLL}$ threshold corrections are given by

$$
\begin{aligned}
\hat{\sigma}_{q \bar{q} \rightarrow Q \bar{Q}}^{\overline{\mathrm{MS}}(3)}\left(s_{4}, m^{2}, s, t_{1}, u_{1}, \mu_{F}, \mu_{R}\right)=\sigma_{q \bar{q} \rightarrow Q \bar{Q}}^{B}\left(\frac{\alpha_{s}\left(\mu_{R}^{2}\right)}{\pi}\right)^{3}\left\{8 C_{F}^{3}\left[\frac{\ln ^{5}\left(s_{4} / m^{2}\right)}{s_{4}}\right]_{+}\right. \\
+20 C_{F}^{2}\left[\operatorname{Re}_{22}^{\prime q \bar{q}}-C_{F}+C_{F} \ln \left(\frac{s m^{2}}{t_{1} u_{1}}\right)-C_{F} \ln \left(\frac{\mu_{F}^{2}}{m^{2}}\right)-\frac{\beta_{0}}{6}\right]\left[\frac{\ln ^{4}\left(s_{4} / m^{2}\right)}{s_{4}}\right]_{+} \\
+\left[8 C_{F}^{2} c_{1}+4 C_{F} c_{2}^{2}+16 C_{F} \Gamma_{12}^{q \bar{q}} \Gamma_{21}^{\prime q \bar{q}}+\frac{\beta_{0}^{2}}{3} C_{F}-64 C_{F}^{3} \zeta_{2}+8 C_{F}^{2} K\right. \\
\left.\left.+4 \beta_{0} C_{F}\left(-\frac{c_{2}}{3}+C_{F} \ln \left(\frac{t_{1} u_{1}}{s m^{2}}\right)+C_{F} \ln \left(\frac{\mu_{R}^{2}}{m^{2}}\right)+C_{F}-\operatorname{Re}_{22}^{\prime q \bar{q}}\right)\right]\left[\frac{\ln ^{3}\left(s_{4} / m^{2}\right)}{s_{4}}\right]_{+}\right\} \\
\quad+\mathcal{O}\left(\left[\frac{\left.\ln ^{2}\left(s_{4}\right) / m^{2}\right)}{s_{4}}\right]_{+}\right)
\end{aligned}
$$

where $c_{1}, c_{2}$ are defined below Eq. (3.14). Note that at NNLL accuracy there are no cubic terms in $\Gamma_{S}$; they start contributing at $\mathcal{O}\left(\left[\left(\ln ^{2}\left(s_{4}\right) / m^{2}\right) / s_{4}\right]_{+}\right)$. One can also derive terms involving the factorization and renormalization scales at lower powers of the logarithms as was explained in Section 3.

For the $g g$ channel the leading logarithms at $\mathrm{N}^{3} \mathrm{LO}$ are of course the same as for the $q \bar{q}$ channel with the substitution $C_{F} \rightarrow C_{A}$ in the coefficients. Beyond leading logarithms the more complex color structure of the hard scattering for the $g g$ channel makes the calculation more lengthy, as is evident already at NNLO, and will not be pursued here.

In the DIS scheme the corresponding result is

$$
\begin{aligned}
\hat{\sigma}_{q \bar{q} \rightarrow Q \bar{Q}}^{\mathrm{DIS}(3)}\left(s_{4}, m^{2}, s, t_{1}, u_{1}, \mu_{F}, \mu_{R}\right)=\sigma_{q \bar{q} \rightarrow Q \bar{Q}}^{B}\left(\frac{\alpha_{s}\left(\mu_{R}^{2}\right)}{\pi}\right)^{3}\left\{C_{F}^{3}\left[\frac{\ln ^{5}\left(s_{4} / m^{2}\right)}{s_{4}}\right]_{+}\right. \\
+5 C_{F}^{2}\left[\operatorname{Re}_{22}^{\prime q \bar{q}}-\frac{C_{F}}{4}+\frac{1}{2} C_{F} \ln \left(\frac{s^{2}}{t_{1} u_{1}}\right)-C_{F} \ln \left(\frac{\mu_{F}^{2}}{m^{2}}\right)-\frac{\beta_{0}}{4}\right]\left[\frac{\ln ^{4}\left(s_{4} / m^{2}\right)}{s_{4}}\right]_{+} \\
\quad+\left[2 C_{F}^{2} c_{1}^{\prime}+2 C_{F} c_{2}^{\prime 2}+8 C_{F} \Gamma_{12}^{\prime q \bar{q}} \Gamma_{21}^{\prime q \bar{q}}+\frac{7}{24} \beta_{0}^{2} C_{F}-8 C_{F}^{3} \zeta_{2}+2 C_{F}^{2} K\right. \\
\left.\left.\quad+\beta_{0} C_{F}\left(-c_{2}^{\prime}+\frac{3}{2} C_{F} \ln \left(\frac{t_{1} u_{1}}{m^{4}}\right)+C_{F} \ln \left(\frac{\mu_{R}^{2}}{s}\right)+\frac{5}{4} C_{F}-2 \operatorname{Re} \Gamma_{22}^{\prime q \bar{q}}\right)\right]\left[\frac{\ln ^{3}\left(s_{4} / m^{2}\right)}{s_{4}}\right]_{+}\right\} \\
\quad+\mathcal{O}\left(\left[\frac{\left.\ln ^{2}\left(s_{4}\right) / m^{2}\right)}{s_{4}}\right]_{+}\right)
\end{aligned}
$$

where $c_{1}^{\prime}=c_{\text {DIS }}^{(1) q \bar{q} \mathrm{~S}+\mathrm{V}}$, and $c_{2}^{\prime}=2 \operatorname{Re} \Gamma_{22}^{\prime q \bar{q}}-C_{F} / 2+C_{F} \ln \left(s^{2} /\left(t_{1} u_{1}\right)\right)-2 C_{F} \ln \left(\mu_{F}^{2} / m^{2}\right)$.

We can extend our study of subleading logarithms to $\mathrm{N}^{3} \mathrm{LO}$. The $\mathrm{N}^{3} \mathrm{LO}$ threshold $\overline{\mathrm{MS}}$ corrections for $q \bar{q} \rightarrow Q \bar{Q}$ in moment space are given in shorthand notation by

$$
\begin{aligned}
\hat{\sigma}^{(3)}(N)= & \sigma^{B} \frac{\alpha_{s}^{3}}{6 \pi^{3}}\left\{\left[c_{1}-c_{2} \ln \tilde{N}+\frac{c_{3}}{2}\left(\ln ^{2} \tilde{N}+\zeta_{2}\right)\right]^{3}\right. \\
& \left.+\tilde{F}^{\prime}\left(\beta_{0}, \Gamma_{S}^{2}, \Gamma_{S}^{3}, K, 3-\operatorname{loop}\right)\right\}
\end{aligned}
$$

i.e. by the cube of the terms in curly brackets in Eq. (3.15) plus a function $\tilde{F}^{\prime}$ that gives the $\beta_{0}$ terms that come from changing the argument in the running coupling, the two-loop $K$ 
terms, additional square and cubic terms in the soft anomalous dimension matrix elements, and two- and three-loop $\Gamma_{S}$ and other terms. Note that we have also absorbed in $\tilde{F}^{\prime}$ the terms $2 T_{1}^{3}-3 T_{1}^{2}\left[c_{1}-c_{2} \ln \tilde{N}+\left(c_{3} / 2\right)\left(\ln ^{2} \tilde{N}+\zeta_{2}\right)\right]$, with $T_{1}=c_{1}(\mu=m)$. We then rewrite Eq. (4.3) in terms of $I_{5}, I_{4}, I_{3}, I_{2}, I_{1}$, and $I_{0}$ defined in Appendix A.

Inverting back to momentum space, we have

$$
\begin{aligned}
\hat{\sigma}^{(3)}\left(s_{4}\right)= & \sigma^{B} \frac{\alpha_{s}^{3}}{\pi^{3}}\left\{\frac{1}{8} c_{3}^{3}\left[\frac{\ln ^{5}\left(s_{4} / m^{2}\right)}{s_{4}}\right]_{+}+\frac{5}{8} c_{3}^{2} c_{2}\left[\frac{\ln ^{4}\left(s_{4} / m^{2}\right)}{s_{4}}\right]_{+}\right. \\
& +\left(c_{3} c_{2}^{2}+\frac{c_{1} c_{3}^{2}}{2}-\zeta_{2} c_{3}^{3}\right)\left[\frac{\ln ^{3}\left(s_{4} / m^{2}\right)}{s_{4}}\right]_{+} \\
& +\left(\frac{3}{2} c_{1} c_{2} c_{3}-3 \zeta_{2} c_{3}^{2} c_{2}+\frac{c_{2}^{3}}{2}+\frac{5}{2} c_{3}^{3} \zeta_{3}\right)\left[\frac{\ln ^{2}\left(s_{4} / m^{2}\right)}{s_{4}}\right]_{+} \\
& +\left[\frac{c_{1}^{2} c_{3}}{2}+c_{1} c_{2}^{2}-\zeta_{2} c_{3}^{2} c_{1}-\frac{5}{2} \zeta_{2} c_{3} c_{2}^{2}+5 \zeta_{3} c_{3}^{2} c_{2}+\frac{5}{4} \zeta_{2}^{2} c_{3}^{3}-\frac{15}{4} c_{3}^{3} \zeta_{4}\right]\left[\frac{\ln \left(s_{4} / m^{2}\right)}{s_{4}}\right]_{+} \\
& +\left[\frac{1}{2} c_{2} c_{1}^{2}+3 c_{3}^{3} \zeta_{5}-\frac{15}{4} c_{3}^{2} c_{2} \zeta_{4}-2 c_{3}^{3} \zeta_{2} \zeta_{3}+c_{1} c_{3}^{2} \zeta_{3}+2 c_{3} c_{2}^{2} \zeta_{3}\right. \\
& \left.+\frac{5}{4} c_{3}^{2} c_{2} \zeta_{2}^{2}-c_{1} c_{2} c_{3} \zeta_{2}-\frac{1}{2} \zeta_{2} c_{2}^{3}\right]\left[\frac{1}{s_{4}}\right]+ \\
& +\left[\frac{c_{1}^{3}}{6}-\frac{5}{2} c_{3}^{3} \zeta_{6}+3 c_{3}^{2} c_{2} \zeta_{5}+\frac{3}{2} c_{3}^{3} \zeta_{2} \zeta_{4}-\frac{3}{2} c_{3} c_{2}^{2} \zeta_{4}-\frac{3}{4} c_{3}^{2} c_{1} \zeta_{4}\right. \\
& +\frac{5}{6} \zeta_{3}^{2} c_{3}^{3}-2 \zeta_{2} \zeta_{3} c_{2} c_{3}^{2}+c_{1} c_{2} c_{3} \zeta_{3}+\frac{c_{2}^{3}}{3} \zeta_{3}-\frac{1}{6} c_{3}^{3} \zeta_{2}^{3} \\
& \left.+\frac{1}{4} c_{1} c_{3}^{2} \zeta_{2}^{2}+\frac{1}{2} c_{3} c_{2}^{2} \zeta_{2}^{2}-\frac{1}{2} c_{1} c_{2}^{2} \zeta_{2}\right] \delta\left(s_{4}\right) \\
& \left.+F^{\prime}\left(\beta_{0}, \Gamma_{S}^{2}, \Gamma_{S}^{3}, K, 3-\operatorname{loop}\right)\right\}
\end{aligned}
$$

where $F^{\prime}$ comes from the inversion of $\tilde{F}^{\prime}$ and starts contributing at NLL and higher accuracy. One can easily see that the above equation, with the appropriate explicit expression for $F^{\prime}$, agrees with the $\mathrm{N}^{3} \mathrm{LO}-\mathrm{NNLL}$ expansion given in Eq. (4.1). Of course we cannot derive all of the $\left[\left(\ln ^{2}\left(s_{4} / m^{2}\right)\right) / s_{4}\right]_{+}$and lower terms in Eq. (4.4) because of unknown 3-loop corrections in $F^{\prime}$.

Again, let us see what happens if one keeps the logarithms only at a certain accuracy. At NLL accuracy for $\hat{\sigma}^{(3)}$ the subleading terms from the inversion (keeping only $\zeta$ terms and no $\beta_{0}, K, \gamma_{E}$ terms as discussed in Section 3.3) are:

$$
\begin{gathered}
\sigma^{B} \frac{\alpha_{s}^{3}}{\pi^{3}}\left\{-\frac{5}{4} \zeta_{2} c_{3}^{3}\left[\frac{\ln ^{3}\left(s_{4} / m^{2}\right)}{s_{4}}\right]_{+}+\left(\frac{5}{2} \zeta_{3} c_{3}^{3}-\frac{15}{4} c_{3}^{2} c_{2} \zeta_{2}\right)\left[\frac{\ln ^{2}\left(s_{4} / m^{2}\right)}{s_{4}}\right]_{+}\right. \\
+\left[\frac{15}{8}\left(\zeta_{2}^{2}-2 \zeta_{4}\right) c_{3}^{3}+5 c_{3}^{2} c_{2} \zeta_{3}\right]\left[\frac{\ln \left(s_{4} / m^{2}\right)}{s_{4}}\right]_{+}+\left[\left(-\frac{5}{2} \zeta_{2} \zeta_{3}+3 \zeta_{5}\right) c_{3}^{3}+\frac{15}{8} c_{3}^{2} c_{2}\left(\zeta_{2}^{2}-2 \zeta_{4}\right)\right]\left[\frac{1}{s_{4}}\right]_{+} \\
\left.+\left[\frac{5}{2} c_{3}^{3}\left(-\frac{\zeta_{2}^{3}}{8}+\frac{\zeta_{3}^{2}}{3}+\frac{3}{4} \zeta_{2} \zeta_{4}-\zeta_{6}\right)+c_{3}^{2} c_{2}\left(-\frac{5}{2} \zeta_{2} \zeta_{3}+3 \zeta_{5}\right)\right] \delta\left(s_{4}\right)\right\} .
\end{gathered}
$$

At NNLL accuracy for $\hat{\sigma}^{(3)}$ the corresponding subleading terms from the inversion to mo- 
mentum space are:

$$
\begin{aligned}
& \sigma^{B} \frac{\alpha_{s}^{3}}{\pi^{3}}\left\{\left(\frac{5}{2} \zeta_{3} c_{3}^{3}-\frac{15}{4} c_{3}^{2} c_{2} \zeta_{2}\right)\left[\frac{\ln ^{2}\left(s_{4} / m^{2}\right)}{s_{4}}\right]_{+}\right. \\
& +\left[3\left(-\frac{5}{4} \zeta_{4}+\frac{3}{8} \zeta_{2}^{2}\right) c_{3}^{3}+5 c_{3}^{2} c_{2} \zeta_{3}-3 \zeta_{2} c_{3} c_{2}^{2}-\frac{3}{2} \zeta_{2} c_{1} c_{3}^{2}-48 C_{F} \zeta_{2} \Gamma_{12}^{\prime q \bar{q}} \Gamma_{21}^{\prime q \bar{q}}\right]\left[\frac{\ln \left(s_{4} / m^{2}\right)}{s_{4}}\right]_{+} \\
& +\left[\left(-2 \zeta_{2} \zeta_{3}+3 \zeta_{5}\right) c_{3}^{3}+\frac{15}{8} c_{3}^{2} c_{2}\left(\zeta_{2}^{2}-2 \zeta_{4}\right)+2 \zeta_{3} c_{3} c_{2}^{2}+\zeta_{3} c_{1} c_{3}^{2}+32 C_{F} \zeta_{3} \Gamma_{12}^{\prime q \bar{q}} \Gamma_{21}^{\prime q \bar{q}}\right]\left[\frac{1}{s_{4}}\right]_{+} \\
& +\left[\frac{1}{2} c_{3}^{3}\left(-\frac{1}{4} \zeta_{2}^{3}+\frac{5}{3} \zeta_{3}^{2}+3 \zeta_{2} \zeta_{4}-5 \zeta_{6}\right)+c_{3}^{2} c_{2}\left(-\frac{5}{2} \zeta_{2} \zeta_{3}+3 \zeta_{5}\right)\right. \\
& \left.\left.+\frac{3}{4}\left(c_{3} c_{2}^{2}+\frac{c_{1} c_{3}^{2}}{2}\right)\left(\zeta_{2}^{2}-2 \zeta_{4}\right)+12 C_{F} \Gamma_{12}^{\prime q \bar{q}} \Gamma_{21}^{\prime q \bar{q}}\left(\zeta_{2}^{2}-2 \zeta_{4}\right)\right] \delta\left(s_{4}\right)\right\}
\end{aligned}
$$

A comparison of both the NLL and the NNLL subleading terms with Eq. (4.4) shows that most of these terms have incorrect coefficients. The subleading terms bring down the value of the $\overline{\mathrm{MS}} \mathrm{N}^{3} \mathrm{LO}$ corrections for $q \bar{q} \rightarrow t \bar{t}$ at the Tevatron with $\sqrt{S}=1.8 \mathrm{TeV}$ and $m=175 \mathrm{GeV} / c^{2}$ from $0.9 \mathrm{pb}(1.1 \mathrm{pb})$ at NNLL (NLL) to around $0.3 \mathrm{pb}$. As we discussed in Section 3.3, retaining subleading terms with incorrect coefficients in the expansions can produce misleading results. We also note that in the DIS scheme the corresponding corrections (without subleading terms) are $0.2 \mathrm{pb}$, again smaller than the $\overline{\mathrm{MS}}$ result because of the specification of the DIS scheme.

Since we don't know the (potentially large) coefficients of subleading powers (beyond NNLL) of the logarithms at $\mathrm{N}^{3} \mathrm{LO}$, in contrast to the NNLO calculation where all logarithms were determined, we cannot make firm numerical predictions at this order. This also relates to the questions raised in Ref. [40] as we discussed earlier. A full $\mathrm{N}^{3} \mathrm{LO}$ calculation may give substantially different (and smaller) results from the $\mathrm{N}^{3} \mathrm{LO}-\mathrm{NNLL}$ calculation; but at present we cannot calculate corrections beyond NNLL accuracy. Therefore, as we have stated before, for reliable numerical predictions we prefer to stop the expansion at NNLO.

\section{$5 \quad \mathrm{~N}^{4} \mathrm{LO}$ and higher-order threshold corrections}

Finally, we briefly discuss the corrections at next-to-next-to-next-to-next-to-leading and higher orders.

For the $q \bar{q}$ channel in the $\overline{\mathrm{MS}}$ scheme at NLL accuracy, the $\mathrm{N}^{4} \mathrm{LO}$ threshold corrections are given by

$$
\begin{aligned}
& \hat{\sigma}_{q \bar{q} \rightarrow Q \bar{Q}}^{\overline{\mathrm{MS}}(4)}\left(s_{4}, m^{2}, s, t_{1}, u_{1}, \mu_{F}, \mu_{R}\right)=\sigma_{q \bar{q} \rightarrow Q \bar{Q}}^{B}\left(\frac{\alpha_{s}\left(\mu_{R}^{2}\right)}{\pi}\right)^{4}\left\{\frac{16}{3} C_{F}^{4}\left[\frac{\ln ^{7}\left(s_{4} / m^{2}\right)}{s_{4}}\right]_{+}\right. \\
& \left.\quad+\frac{56}{3} C_{F}^{3}\left[\operatorname{Re}_{22}^{\prime q \bar{q}}-C_{F}+C_{F} \ln \left(\frac{s m^{2}}{t_{1} u_{1}}\right)-C_{F} \ln \left(\frac{\mu_{F}^{2}}{m^{2}}\right)-\frac{\beta_{0}}{4}\right]\left[\frac{\ln ^{6}\left(s_{4} / m^{2}\right)}{s_{4}}\right]_{+}\right\} \\
& \quad+\mathcal{O}\left(\left[\frac{\left.\ln ^{5}\left(s_{4}\right) / m^{2}\right)}{s_{4}}\right]_{+}\right) .
\end{aligned}
$$

By matching with the exact NLO cross section, as we have described before, one can derive the full NNLL terms as well. We note that no cubic or quartic terms in $\Gamma_{S}$ appear at NNLL accuracy. 
A full determination of the cross section at this order would require four-loop calculations. The leading logarithms for the $g g$ channel at $\mathrm{N}^{4} \mathrm{LO}$ again follow from Eq. (5.1) with the substitution $C_{F} \rightarrow C_{A}$.

In the DIS scheme the corresponding NLL result is

$$
\begin{aligned}
& \hat{\sigma}_{q \bar{q} \rightarrow Q \bar{Q}}^{\mathrm{DIS}(4)}\left(s_{4}, m^{2}, s, t_{1}, u_{1}, \mu_{F}, \mu_{R}\right)=\sigma_{q \bar{q} \rightarrow Q \bar{Q}}^{B}\left(\frac{\alpha_{s}\left(\mu_{R}^{2}\right)}{\pi}\right)^{4}\left\{\frac{1}{3} C_{F}^{4}\left[\frac{\ln ^{7}\left(s_{4} / m^{2}\right)}{s_{4}}\right]_{+}\right. \\
& \left.\quad+\frac{7}{3} C_{F}^{3}\left[\operatorname{Re} \Gamma_{22}^{\prime q \bar{q}}-\frac{C_{F}}{4}+\frac{1}{2} C_{F} \ln \left(\frac{s^{2}}{t_{1} u_{1}}\right)-C_{F} \ln \left(\frac{\mu_{F}^{2}}{m^{2}}\right)-\frac{15}{56} \beta_{0}\right]\left[\frac{\ln ^{6}\left(s_{4} / m^{2}\right)}{s_{4}}\right]_{+}\right\} \\
& \quad+\mathcal{O}\left(\left[\frac{\left.\ln ^{5}\left(s_{4}\right) / m^{2}\right)}{s_{4}}\right]_{+}\right) .
\end{aligned}
$$

Again, numerically the corrections in the DIS scheme are smaller than in the $\overline{\mathrm{MS}}$ scheme.

The finite-order expansion procedure can be extended to arbitrarily high orders at NNLL accuracy. In general, at $n$th order in $\alpha_{s}$ (beyond the Born term) the leading logarithms in the $\overline{\mathrm{MS}}$ scheme are

$$
\hat{\sigma}_{q \bar{q} \rightarrow Q \bar{Q}}^{\overline{\mathrm{MS}}(n)}\left(s_{4}, m^{2}, s, t_{1}, u_{1}, \mu_{F}, \mu_{R}\right)=\sigma_{q \bar{q} \rightarrow Q \bar{Q}}^{B}\left(\frac{\alpha_{s}\left(\mu_{R}^{2}\right)}{\pi}\right)^{n} \frac{2 n}{n !}\left(2 C_{F}\right)^{n}\left[\frac{\ln ^{2 n-1}\left(s_{4} / m^{2}\right)}{s_{4}}\right]_{+}+\ldots
$$

For the DIS scheme we only need to replace $\left(2 C_{F}\right)^{n}$ by $C_{F}^{n}$ in the above equation. For the $g g$ channel we simply replace $C_{F}$ by $C_{A}$ as discussed before. It is easy to check that Eq. (5.3) reproduces the leading logarithms in all the expansions presented in this paper.

\section{Conclusions}

Threshold resummation can make powerful improvements to NLO calculations of heavy quark production cross sections. The analytical form of the threshold corrections to the fully differential cross section can be derived at NNLL accuracy at arbitrarily high order and explicit results have been provided in this paper through $\mathrm{N}^{4} \mathrm{LO}$. The role of subleading logarithms has been studied in detail and it has been shown that care must be taken to arrive at reliable numerical predictions for the cross section. For top quark production at the Tevatron NNLO-NNLL predictions have been made for the total cross section and transverse momentum distributions. The NNLO-NNLL corrections are significant and they dramatically reduce the dependence of the cross section on the scale relative to NLO.

\section{Acknowledgements}

I wish to thank George Sterman for drawing my attention to the importance of subleading logarithms in resummation prescriptions. I would also like to thank Eric Laenen, Jeff Owens, Jack Smith, and Ramona Vogt for many discussions. Some of the results in sections 3.2 and 3.4 were derived within the collaboration of Ref. [19] and will also be discussed along with other results in that paper. This work was supported in part by the U.S. Department of Energy. 


\section{A Mellin transforms}

Here we present some useful formulas for the Mellin transforms that are used in the resummed cross section and the finite-order expansions.

We define

$$
I_{n}(N)=\int_{0}^{1} d z z^{N-1}\left[\frac{\ln ^{n}(1-z)}{1-z}\right]_{+} .
$$

One may also define

$$
I_{n}(N)=\int_{0}^{\infty} d s_{4} e^{-N s_{4} / m^{2}}\left[\frac{\ln ^{n}\left(s_{4} / m^{2}\right)}{s_{4}}\right]_{+} .
$$

The results below are identical for either definition.

Then, we have [2]

$$
\left.I_{n}(N)\right|_{N \rightarrow+\infty}=\lim _{\epsilon \rightarrow 0^{+}}\left(\frac{\partial}{\partial \epsilon}\right)^{n}\left[\frac{1}{\epsilon}\left(e^{\epsilon \alpha(\epsilon)}-1\right)\right]\left[1+O\left(\frac{1}{N}\right)\right]
$$

where

$$
\alpha(\epsilon)=-\ln \tilde{N}+\sum_{n=2}^{\infty}(-1)^{n} \frac{\epsilon^{n-1}}{n} \zeta_{n}=\sum_{n=0}^{\infty} a_{n} \epsilon^{n}
$$

with

$$
a_{0}=-\ln \tilde{N}, \quad a_{i}=\frac{(-1)^{i+1}}{i+1} \zeta_{i+1}, \quad i=1, \ldots, \infty .
$$

Here $\tilde{N}=N \mathrm{e}^{\gamma_{E}}$ with $\gamma_{E}$ the Euler constant, $\gamma_{E}=0.577 \ldots$ Also $\zeta_{2}=\pi^{2} / 6, \zeta_{4}=\pi^{4} / 90, \zeta_{6}=$ $\pi^{6} / 945, \zeta_{8}=\pi^{8} / 9450$, etc., while $\zeta_{3}=1.2020569 \ldots, \zeta_{5}=1.0369278 \ldots, \zeta_{7}=1.0083493 \ldots$, etc.

Then

$$
\begin{aligned}
I_{n}(N)= & \frac{a_{0}^{n+1}}{n+1}+n a_{0}^{n-1} a_{1}+n(n-1) a_{0}^{n-2} a_{2}+n(n-1)(n-2) a_{0}^{n-3}\left(\frac{a_{1}^{2}}{2}+a_{3}\right) \\
& +n(n-1)(n-2)(n-3) a_{0}^{n-4}\left(a_{1} a_{2}+a_{4}\right) \\
& +n(n-1)(n-2)(n-3)(n-4) a_{0}^{n-5}\left(\frac{a_{1}^{3}}{6}+\frac{a_{2}^{2}}{2}+a_{1} a_{3}+a_{5}\right)+\ldots
\end{aligned}
$$

The expressions for $I_{n}$ have been presented up to $n=3$ in [2]. Here we extend this table to $n=7$, useful through $\mathrm{N}^{4} \mathrm{LO}$ expansions:

$$
\begin{aligned}
& I_{0}(N)=-\ln \tilde{N} \\
& I_{1}(N)=\frac{1}{2} \ln ^{2} \tilde{N}+\frac{\zeta_{2}}{2} \\
& I_{2}(N)=-\frac{1}{3} \ln ^{3} \tilde{N}-\zeta_{2} \ln \tilde{N}-\frac{2}{3} \zeta_{3} \\
& I_{3}(N)=\frac{1}{4} \ln ^{4} \tilde{N}+\frac{3}{2} \zeta_{2} \ln ^{2} \tilde{N}+2 \zeta_{3} \ln \tilde{N}+\frac{3}{2} \zeta_{4}+\frac{3}{4} \zeta_{2}^{2} \\
& I_{4}(N)=-\frac{1}{5} \ln ^{5} \tilde{N}-2 \zeta_{2} \ln ^{3} \tilde{N}-4 \zeta_{3} \ln ^{2} \tilde{N}-3 \ln \tilde{N}\left(\zeta_{2}^{2}+2 \zeta_{4}\right)-4\left(\zeta_{2} \zeta_{3}+\frac{6}{5} \zeta_{5}\right) \\
& I_{5}(N)=\frac{1}{6} \ln ^{6} \tilde{N}+\frac{5}{2} \zeta_{2} \ln ^{4} \tilde{N}+\frac{20}{3} \zeta_{3} \ln ^{3} \tilde{N}+\frac{15}{2}\left(\zeta_{2}^{2}+2 \zeta_{4}\right) \ln ^{2} \tilde{N}
\end{aligned}
$$




$$
\begin{aligned}
& +4\left(5 \zeta_{2} \zeta_{3}+6 \zeta_{5}\right) \ln \tilde{N}+5\left(\frac{\zeta_{2}^{3}}{2}+\frac{4}{3} \zeta_{3}^{2}+3 \zeta_{2} \zeta_{4}+4 \zeta_{6}\right) \\
I_{6}(N)= & -\frac{1}{7} \ln ^{7} \tilde{N}-3 \zeta_{2} \ln ^{5} \tilde{N}-10 \zeta_{3} \ln ^{4} \tilde{N}-15\left(\zeta_{2}^{2}+2 \zeta_{4}\right) \ln ^{3} \tilde{N}-12\left(6 \zeta_{5}+5 \zeta_{2} \zeta_{3}\right) \ln ^{2} \tilde{N} \\
& -15\left(8 \zeta_{6}+\zeta_{2}^{3}+\frac{8}{3} \zeta_{3}^{2}+6 \zeta_{2} \zeta_{4}\right) \ln \tilde{N}-60 \zeta_{3} \zeta_{4}-\frac{720}{7} \zeta_{7}-72 \zeta_{2} \zeta_{5}-30 \zeta_{2}^{2} \zeta_{3} \\
I_{7}(N)= & \frac{1}{8} \ln ^{8} \tilde{N}+\frac{7}{2} \zeta_{2} \ln ^{6} \tilde{N}+14 \zeta_{3} \ln ^{5} \tilde{N}+\frac{105}{4}\left(\zeta_{2}^{2}+2 \zeta_{4}\right) \ln ^{4} \tilde{N}+4\left(42 \zeta_{5}+35 \zeta_{2} \zeta_{3}\right) \ln ^{3} \tilde{N} \\
+\frac{105}{2}\left(\zeta_{2}^{3}+\right. & \left.8 \zeta_{6}+\frac{8}{3} \zeta_{3}^{2}+6 \zeta_{2} \zeta_{4}\right) \ln ^{2} \tilde{N}+4\left(126 \zeta_{2} \zeta_{5}+105 \zeta_{3} \zeta_{4}+180 \zeta_{7}+\frac{105}{2} \zeta_{2}^{2} \zeta_{3}\right) \ln \tilde{N} \\
& +\frac{105}{8} \zeta_{2}^{4}+336 \zeta_{3} \zeta_{5}+\frac{315}{2} \zeta_{4}\left(\zeta_{2}^{2}+\zeta_{4}\right)+420 \zeta_{2} \zeta_{6}+630 \zeta_{8}+140 \zeta_{2} \zeta_{3}^{2} .
\end{aligned}
$$

\section{B Hadronic cross sections}

The double differential hadronic cross section $d^{2} \sigma_{h_{1} h_{2}} / d T_{1} d U_{1}$ is written as a convolution of parton distributions with the partonic differential cross section:

$$
S^{2} \frac{d^{2} \sigma_{h_{1} h_{2}}\left(S, T_{1}, U_{1}\right)}{d T_{1} d U_{1}}=\sum_{i, j} \int_{x_{1}^{-}}^{1} \frac{d x_{1}}{x_{1}} \int_{x_{2}^{-}}^{1} \frac{d x_{2}}{x_{2}} \phi_{i / h_{1}}\left(x_{1}, \mu_{F}^{2}\right) \phi_{j / h_{2}}\left(x_{2}, \mu_{F}^{2}\right) s^{2} \frac{d^{2} \hat{\sigma}_{i j}\left(s, t_{1}, u_{1}\right)}{d t_{1} d u_{1}}
$$

where the sum is over all massless parton flavors and $\phi_{i}\left(x, \mu_{F}^{2}\right)$ are the parton distribution functions for flavor $i$ in hadron $h$ at factorization scale $\mu_{F}$. The hadronic invariants $S, T_{1}, U_{1}$ are defined in analogy to their partonic counterparts. The lower limits of the momentum fractions of the partons in the hadrons are given by $x_{1}^{-}=-U_{1} /\left(S+T_{1}\right)$ and $x_{2}^{-}=-x_{1} T_{1} /\left(x_{1} S+U_{1}\right)$.

By making a transformation from the variables $\left(T_{1}, U_{1}, x_{1}, x_{2}\right)$ to the variables $\left(Y, p_{T}^{2}, x_{1}, s_{4}\right)$, with $Y$ and $p_{T}$ the rapidity and transverse momentum, via

$$
T_{1}=\sqrt{S}\left(p_{T}^{2}+m^{2}\right) e^{-Y}, \quad U_{1}=\sqrt{S}\left(p_{T}^{2}+m^{2}\right) e^{Y}, \quad x_{2}=\frac{s_{4}-x_{1} T_{1}}{x_{1} S+U_{1}},
$$

we may write the differential cross section in $p_{T}$ and $Y$ as

$$
\frac{d^{2} \sigma_{h_{1} h_{2}}}{d p_{T}^{2} d Y}=\sum_{i j} \frac{1}{S} \int_{x_{1}^{-}}^{1} \frac{d x_{1}}{x_{1}} \int_{0}^{x_{1}\left(S+T_{1}\right)+U_{1}} \frac{d s_{4}}{s_{4}-x_{1} T_{1}} \phi\left(x_{1}\right) \phi\left(\frac{s_{4}-x_{1} T_{1}}{x_{1} S+U_{1}}\right) s^{2} \frac{d^{2} \hat{\sigma}_{i j}}{d t_{1} d u_{1}} .
$$

Now, let us write the $k$ th-order partonic threshold corrections in the shorthand notation

$$
s^{2} \frac{d^{2} \hat{\sigma}_{i j}^{(k)}\left(s, t_{1}, u_{1}\right)}{d t_{1} d u_{1}}=\left(\frac{\alpha_{s}}{\pi}\right)^{k}\left\{A^{i j}\left(s, t_{1}, u_{1}\right) \delta\left(s_{4}\right)+\sum_{l=0}^{2 k-1} a_{l}^{i j}\left(s, t_{1}, u_{1}\right)\left[\frac{\ln ^{l}\left(s_{4} / m^{2}\right)}{s_{4}}\right]_{+}\right\} \text {. }
$$

By substituting the above expression for the partonic threshold corrections in Eq. (B.3), we can write the hadronic $k$ th-order corrections as

$$
\begin{aligned}
\frac{d^{2} \sigma_{h_{1} h_{2}}^{(k)}}{d p_{T}^{2} d Y}= & \sum_{i j}\left(\frac{\alpha_{s}}{\pi}\right)^{k} \frac{1}{S} \int_{x_{1}^{-}}^{1} \frac{d x_{1}}{x_{1}} \phi\left(x_{1}\right) \int_{0}^{x_{1}\left(S+T_{1}\right)+U_{1}} \frac{d s_{4}}{s_{4}-x_{1} T_{1}} \phi\left(\frac{s_{4}-x_{1} T_{1}}{x_{1} S+U_{1}}\right) \\
& \times\left\{A^{i j}\left(s_{4}\right) \delta\left(s_{4}\right)+\sum_{l=0}^{2 k-1} a_{l}^{i j}\left(s_{4}\right)\left[\frac{\ln ^{l}\left(s_{4} / m^{2}\right)}{s_{4}}\right]_{+}\right\} .
\end{aligned}
$$


After some rearrangements of terms and partial integrations, we can rewrite this as

$$
\begin{gathered}
\frac{d^{2} \sigma_{h_{1} h_{2}}^{(k)}}{d p_{T}^{2} d Y}=\sum_{i j}\left(\frac{\alpha_{s}}{\pi}\right)^{k} \frac{1}{S} \sum_{l=0}^{2 k-1}\left\{\int_{x_{1}^{-}}^{1} \frac{d x_{1}}{x_{1}} \phi\left(x_{1}\right) \int_{0}^{x_{1}\left(S+T_{1}\right)+U_{1}} d s_{4} \theta\left(s_{4}-\Delta\right)\right. \\
\times \frac{1}{s_{4}} \ln ^{l}\left(\frac{s_{4}}{m^{2}}\right)\left[a_{l}\left(s_{4}\right) \frac{1}{s_{4}-x_{1} T_{1}} \phi\left(\frac{s_{4}-x_{1} T_{1}}{x_{1} S+U_{1}}\right)-a_{l}(0) \frac{1}{\left(-x_{1} T_{1}\right)} \phi\left(\frac{-x_{1} T_{1}}{x_{1} S+U_{1}}\right)\right] \\
\left.+\int_{x_{1}^{-}}^{1} \frac{d x_{1}}{x_{1}}\left[\frac{1}{l+1} \ln ^{l+1}\left(\frac{x_{1}\left(S+T_{1}\right)+U_{1}}{m^{2}}\right) a_{l}(0)+A(0)\right] \frac{1}{\left(-x_{1} T_{1}\right)} \phi\left(x_{1}\right) \phi\left(\frac{-x_{1} T_{1}}{x_{1} S+U_{1}}\right)\right\} .
\end{gathered}
$$

The transverse momentum distributions are then given by

$$
\frac{d \sigma_{h_{1} h_{2}}^{(k)}}{d p_{T}}=2 p_{T} \frac{d \sigma_{h_{1} h_{2}}^{(k)}}{d p_{T}^{2}}=2 p_{T} \int_{Y^{-}}^{Y^{+}} d Y \frac{d^{2} \sigma_{h_{1} h_{2}}^{(k)}}{d p_{T}^{2} d Y}
$$

where

$$
Y^{ \pm}= \pm \frac{1}{2} \ln \left(\frac{1+\beta_{T}}{1-\beta_{T}}\right)
$$

and $\beta_{T}=\sqrt{1-4\left(p_{T}^{2}+m^{2}\right) / S}$. The total cross section can then be retrieved by integrating Eq. (B.7) over $p_{T}$ with lower limit 0 and upper limit $\sqrt{S / 4-m^{2}}$.

\section{NLO and NNLO threshold corrections in PIM kine- matics}

In this Appendix we present results for the NLO and NNLO expansions of the resummed cross section in heavy-quark-pair inclusive kinematics (see also Refs. [7, 13, 19]). Here the distance from threshold is measured in terms of the variable $z=Q^{2} / s$, with $Q^{2}$ the invariant mass squared of the heavy quark-antiquark pair, and the corresponding "plus" distributions are of the form $\left[\left(\ln ^{k}(1-z)\right) /(1-z)\right]_{+}$.

\section{C.1 NLO threshold corrections}

In the $\overline{\mathrm{MS}}$ scheme the NLO-NLL corrections for the $q \bar{q} \rightarrow Q \bar{Q}$ channel are

$$
\begin{aligned}
\hat{\sigma}_{q \bar{q} \rightarrow Q \bar{Q}}^{\overline{\mathrm{MS}}(1)}(1 & \left.-z, m^{2}, s, t_{1}, u_{1}, \mu_{F}, \mu_{R}\right)=\sigma_{q \bar{q} \rightarrow Q \bar{Q}}^{B} \frac{\alpha_{s}\left(\mu_{R}^{2}\right)}{\pi}\left\{4 C_{F}\left[\frac{\ln (1-z)}{1-z}\right]_{+}\right. \\
+ & {\left[\frac{1}{1-z}\right]_{+}\left[2 \operatorname{Re} \Gamma_{22}^{q \bar{q}}-2 C_{F}-2 C_{F} \ln \left(\frac{\mu_{F}^{2}}{s}\right)\right] } \\
& \left.+\delta(1-z)\left[-\frac{3}{2} C_{F} \ln \left(\frac{\mu_{F}^{2}}{s}\right)+\frac{\beta_{0}}{2} \ln \left(\frac{\mu_{R}^{2}}{s}\right)\right]\right\} .
\end{aligned}
$$

We note that in the $\delta(1-z)$ contribution the expansion reproduces only the scale-dependent terms. The rest of the $\delta(1-z)$ terms can only be obtained by matching to the exact NLO cross section in PIM kinematics [19]; this is exactly analogous to what was presented in Section 3 for 1PI kinematics. 
In the DIS scheme the corresponding result is

$$
\begin{aligned}
\hat{\sigma}_{q \bar{q} \rightarrow Q \bar{Q}}^{\operatorname{DIS}(1)}(1 & \left.-z, m^{2}, s, t_{1}, u_{1}, \mu_{F}, \mu_{R}\right)=\sigma_{q \bar{q} \rightarrow Q \bar{Q}}^{B} \frac{\alpha_{s}\left(\mu_{R}^{2}\right)}{\pi}\left\{2 C_{F}\left[\frac{\ln (1-z)}{1-z}\right]_{+}\right. \\
+ & {\left[\frac{1}{1-z}\right]_{+}\left[2 \operatorname{Re} \Gamma_{22}^{q \bar{q}}-\frac{C_{F}}{2}-2 C_{F} \ln \left(\frac{\mu_{F}^{2}}{s}\right)\right] } \\
& \left.+\delta(1-z)\left[-\frac{3}{2} C_{F} \ln \left(\frac{\mu_{F}^{2}}{s}\right)+\frac{\beta_{0}}{2} \ln \left(\frac{\mu_{R}^{2}}{s}\right)\right]\right\} .
\end{aligned}
$$

For the $g g \rightarrow Q \bar{Q}$ channel the NLO-NLL corrections in the $\overline{\mathrm{MS}}$ scheme are

$$
\begin{aligned}
& \hat{\sigma}_{g g \rightarrow Q \bar{Q}}^{\overline{\mathrm{MS}}(1)}\left(1-z, m^{2}, s, t_{1}, u_{1}, \mu_{F}, \mu_{R}\right)=\sigma_{g g \rightarrow Q \bar{Q}}^{B} \frac{\alpha_{s}\left(\mu_{R}^{2}\right)}{\pi}\left\{4 C_{A}\left[\frac{\ln (1-z)}{1-z}\right]_{+}\right. \\
&-\left.2 C_{A} \ln \left(\frac{\mu_{F}^{2}}{s}\right)\left[\frac{1}{1-z}\right]_{+}+\delta(1-z) \frac{\beta_{0}}{2} \ln \left(\frac{\mu_{R}^{2}}{\mu_{F}^{2}}\right)\right\} \\
&+ \alpha_{s}^{3}\left(\mu_{R}^{2}\right) K_{g g} B_{Q E D}\left[\frac{1}{1-z}\right]_{+}\left\{N _ { c } ( N _ { c } ^ { 2 } - 1 ) \frac { ( t _ { 1 } ^ { 2 } + u _ { 1 } ^ { 2 } ) } { s ^ { 2 } } \left[\left(-C_{F}+\frac{C_{A}}{2}\right) \operatorname{Re} L_{\beta}\right.\right. \\
&\left.+\frac{C_{A}}{2} \ln \left(\frac{t_{1} u_{1}}{m^{2} s}\right)-C_{F}\right]+\frac{\left(N_{c}^{2}-1\right)}{N_{c}}\left(C_{F}-C_{A}\right) \operatorname{Re} L_{\beta} \\
&\left.-\left(N_{c}^{2}-1\right) \ln \left(\frac{t_{1} u_{1}}{m^{2} s}\right)+C_{F} \frac{\left(N_{c}^{2}-1\right)}{N_{c}}+\frac{N_{c}^{2}}{2}\left(N_{c}^{2}-1\right) \ln \left(\frac{u_{1}}{t_{1}}\right) \frac{\left(t_{1}^{2}-u_{1}^{2}\right)}{s^{2}}\right\} .
\end{aligned}
$$

\section{C.2 NNLO threshold corrections}

We now present the NNLO corrections in PIM kinematics. We give explicit results at NLL accuracy. As noted in Section 3, to reach NNLL accuracy we need to derive matching terms in PIM kinematics [19. The only other difference between the expansions in the two different kinematics are in the extra terms involving $\ln \left(t_{1} u_{1} / m^{4}\right)$ for the 1PI fixed-order expansions relative to the PIM expansions.

In the $\overline{\mathrm{MS}}$ scheme, the NNLO-NLL corrections for $q \bar{q} \rightarrow Q \bar{Q}$ are

$$
\begin{aligned}
\hat{\sigma}_{q \bar{q} \rightarrow Q \bar{Q}}^{\overline{\mathrm{MS}}(2)}(1 & \left.-z, m^{2}, s, t_{1}, u_{1}, \mu_{F}, \mu_{R}\right)=\sigma_{q \bar{q} \rightarrow Q \bar{Q}}^{B}\left(\frac{\alpha_{s}\left(\mu_{R}^{2}\right)}{\pi}\right)^{2}\left\{8 C_{F}^{2}\left[\frac{\ln ^{3}(1-z)}{1-z}\right]_{+}\right. \\
& +\left[\frac{\ln ^{2}(1-z)}{1-z}\right]_{+}\left[-\beta_{0} C_{F}+12 C_{F}\left(\operatorname{Re}_{22}^{\prime q \bar{q}}-C_{F}-C_{F} \ln \left(\frac{\mu_{F}^{2}}{s}\right)\right)\right] \\
& +\left[\frac{\ln (1-z)}{1-z}\right]_{+}\left[C_{F} \ln \left(\frac{\mu_{F}^{2}}{s}\right)\left(-8 \operatorname{Re}_{22}^{q q \bar{q}}+2 C_{F}+4 C_{F} \ln \left(\frac{\mu_{F}^{2}}{s}\right)\right)+3 C_{F} \beta_{0} \ln \left(\frac{\mu_{R}^{2}}{s}\right)\right] \\
& \left.+\left[\frac{1}{1-z}\right]_{+}\left[C_{F}\left(3 C_{F}+\frac{\beta_{0}}{4}\right) \ln ^{2}\left(\frac{\mu_{F}^{2}}{s}\right)-\frac{3}{2} C_{F} \beta_{0} \ln \left(\frac{\mu_{R}^{2}}{s}\right) \ln \left(\frac{\mu_{F}^{2}}{s}\right)\right]\right\} .
\end{aligned}
$$

We note that, at NLL accuracy, in the $[\ln (1-z) /(1-z)]_{+}$coefficient we derive only the terms involving the factorization and renormalization scales, and in the $[1 /(1-z)]_{+}$coefficient we can only determine quadratic terms in the scale logarithms. 
In the DIS scheme the corresponding corrections are

$$
\begin{aligned}
\hat{\sigma}_{q \bar{q} \rightarrow Q \bar{Q}}^{\mathrm{DIS}(2)}(1 & \left.-z, m^{2}, s, t_{1}, u_{1}, \mu_{F}, \mu_{R}\right)=\sigma_{q \bar{q} \rightarrow Q \bar{Q}}^{B}\left(\frac{\alpha_{s}\left(\mu_{R}^{2}\right)}{\pi}\right)^{2}\left\{2 C_{F}^{2}\left[\frac{\ln ^{3}(1-z)}{1-z}\right]_{+}\right. \\
& +\left[\frac{\ln ^{2}(1-z)}{1-z}\right]_{+}\left[-\frac{3 \beta_{0}}{4} C_{F}+6 C_{F}\left(\operatorname{Re}_{22}^{\prime q \bar{q}}-\frac{C_{F}}{4}-C_{F} \ln \left(\frac{\mu_{F}^{2}}{s}\right)\right)\right] \\
& +\left[\frac{\ln (1-z)}{1-z}\right]_{+}\left[C_{F} \ln \left(\frac{\mu_{F}^{2}}{s}\right)\left(-8 \operatorname{Re}_{22}^{\prime q \bar{q}}-C_{F}+4 C_{F} \ln \left(\frac{\mu_{F}^{2}}{s}\right)\right)+\frac{3}{2} C_{F} \beta_{0} \ln \left(\frac{\mu_{R}^{2}}{s}\right)\right] \\
& \left.+\left[\frac{1}{1-z}\right]_{+}\left[C_{F}\left(3 C_{F}+\frac{\beta_{0}}{4}\right) \ln ^{2}\left(\frac{\mu_{F}^{2}}{s}\right)-\frac{3}{2} C_{F} \beta_{0} \ln \left(\frac{\mu_{R}^{2}}{s}\right) \ln \left(\frac{\mu_{F}^{2}}{s}\right)\right]\right\} .
\end{aligned}
$$

In the $\overline{\mathrm{MS}}$ scheme for the $g g \rightarrow Q \bar{Q}$ channel the NNLO-NLL corrections are

$$
\begin{aligned}
\hat{\sigma}_{g g \rightarrow Q \bar{Q}}^{\overline{\mathrm{MS}}(2)}(1- & \left.z, m^{2}, s, t_{1}, u_{1}, \mu_{F}, \mu_{R}\right)=\sigma_{g g \rightarrow Q \bar{Q}}^{B}\left(\frac{\alpha_{s}\left(\mu_{R}^{2}\right)}{\pi}\right)^{2}\left\{8 C_{A}^{2}\left[\frac{\ln ^{3}(1-z)}{1-z}\right]_{+}\right. \\
& \left.+\left[-\beta_{0} C_{A}-12 C_{A}^{2} \ln \left(\frac{\mu_{F}^{2}}{s}\right)\right]\left[\frac{\ln (1-z)}{1-z}\right]_{+}^{2}\right\} \\
+ & \frac{\alpha_{s}^{4}\left(\mu_{R}^{2}\right)}{\pi} K_{g g} B_{\mathrm{QED}}\left[\frac{\ln ^{2}(1-z)}{1-z}\right]_{+} C_{A} 3\left(N_{c}^{2}-1\right)\left\{\frac{\left(t_{1}^{2}+u_{1}^{2}\right)}{s^{2}}\right. \\
& \times\left[N_{c}^{2} \ln \left(\frac{t_{1} u_{1}}{m^{2} s}\right)-2 N_{c}\left(C_{F}-\frac{C_{A}}{2}\right) \operatorname{Re} L_{\beta}-2 N_{c} C_{F}\right]+2 \frac{C_{F}}{N_{c}} \\
& \left.+2 \ln \left(\frac{s m^{2}}{t_{1} u_{1}}\right)+2 \frac{1}{N_{c}}\left(C_{F}-C_{A}\right) \operatorname{Re} L_{\beta}+N_{c}^{2} \frac{\left(t_{1}^{2}-u_{1}^{2}\right)}{s^{2}} \ln \left(\frac{u_{1}}{t_{1}}\right)\right\} \\
+ & \sigma_{g g \rightarrow Q \bar{Q}}^{B}\left(\frac{\alpha_{s}\left(\mu_{R}^{2}\right)}{\pi}\right)^{2}\left[\frac{\ln (1-z)}{1-z}\right]_{+}\left\{\operatorname { l n } ( \frac { \mu _ { F } ^ { 2 } } { s } ) C _ { A } \left[-2 \beta_{0}\right.\right. \\
+ & \frac{\alpha_{s}^{4}\left(\mu_{R}^{2}\right)}{\pi} K_{g g} B_{\mathrm{QED}}\left[\frac{\ln (1-z)}{1-z}\right]_{+} \ln \left(\frac{\mu_{F}^{2}}{s}\right) C_{A} 2\left(N_{c}^{2}-1\right)\left\{N_{c}^{2} \ln \left(\frac{u_{1}}{t_{1}}\right) \frac{\left(u_{1}^{2}-t_{1}^{2}\right)}{s^{2}}\right. \\
+ & 2 N_{c}\left(C_{F}-\frac{C_{A}}{2}\right)\left(\operatorname{Re} L_{\beta}+1\right) \frac{\left(t_{1}^{2}+u_{1}^{2}\right)}{s^{2}}+\ln \left(\frac{t_{1} u_{1}}{s m^{2}}\right) \\
+ & \left.\left.\left.\left.\left(2-\frac{2 C_{F}}{N_{c}}\right)\left(\operatorname{Re} L_{\beta}+1\right)-1\right\}+\frac{\mu_{F}^{2}}{s}\right)\right)+3 C_{A} \beta_{0} \ln \left(\frac{\mu_{R}^{2}}{s}\right)\right\} \\
& \sigma_{g g \rightarrow Q \bar{Q}}^{B}\left(\frac{\alpha_{s}\left(\mu_{R}^{2}\right)}{\pi}\right)^{2}\left[\frac{1}{1-z} C_{A} \beta_{0} \ln { }^{2}\left(\frac{\mu_{F}^{2}}{s}\right)-\frac{3}{2} C_{A} \beta_{0} \ln \left(\frac{\mu_{R}^{2}}{s}\right) \ln \left(\frac{\mu_{F}^{2}}{s}\right)\right] .
\end{aligned}
$$

\section{References}

[1] G. Sterman, Nucl. Phys. B281, 310 (1987).

[2] S. Catani and L. Trentadue, Nucl. Phys. B327, 323 (1989). 
[3] E. Laenen, J. Smith, and W. L. van Neerven, Nucl. Phys. B369, 543 (1992).

[4] E. L. Berger and H. Contopanagos, Phys. Rev. D 54, 3085 (1996).

[5] S. Catani, M. L. Mangano, P. Nason, and L. Trentadue, Nucl. Phys. B478, 273 (1996).

[6] N. Kidonakis and G. Sterman, Phys. Lett. B 387, 867 (1996).

[7] N. Kidonakis and G. Sterman, Nucl. Phys. B505, 321 (1997).

[8] N. Kidonakis, J. Smith, and R. Vogt, Phys. Rev. D 56, 1553 (1997).

[9] N. Kidonakis, Nucl. Phys. B (Proc. Suppl.) 64, 402 (1998).

[10] R. Bonciani, S. Catani, M. L. Mangano, and P. Nason, Nucl. Phys. B529, 424 (1998).

[11] E. Laenen, G. Oderda, and G. Sterman, Phys. Lett. B 438, 173 (1998).

[12] N. Kidonakis and R. Vogt, Phys. Rev. D 59, 074014 (1999).

[13] N. Kidonakis, Int. J. Mod. Phys. A 15, 1245 (2000).

[14] N. Kidonakis, in Corfu 98, JHEP Proceedings, hep-ph/9904507.

[15] H. Contopanagos, E. Laenen, and G. Sterman, Nucl. Phys. B484, 303 (1997).

[16] N. Kidonakis, G. Oderda, and G. Sterman, Nucl. Phys. B525, 299 (1998).

[17] N. Kidonakis, G. Oderda, and G. Sterman, Nucl. Phys. B531, 365 (1998).

[18] E. L. Berger and H. Contopanagos, Phys. Rev. D 57, 253 (1998).

[19] N. Kidonakis, E. Laenen, S. Moch, and R. Vogt, in preparation.

[20] N. Kidonakis and V. Del Duca, Phys. Lett. B 480, 87 (2000).

[21] N. Kidonakis, in Proceedings of the International Europhysics Conference on High Energy Physics, eds. K. Huitu, H. Kurki-Suonio, J. Maalampi (IoP), p. 392, hep-ph/9910240.

[22] N. Kidonakis and J. F. Owens, Phys. Rev. D 61, 094004 (2000).

[23] N. Kidonakis, Nucl. Phys. B (Proc. Suppl). 79, 410 (1999).

[24] N. Kidonakis and J. F. Owens, Phys. Rev. D 63, 054019 (2001).

[25] W. Beenakker, W. L. van Neerven, R. Meng, G. A. Schuler, and J. Smith, Nucl. Phys. B351, 507 (1991).

[26] R. Meng, G. A. Schuler, J. Smith, and W. L. van Neerven, Nucl. Phys. B339, 325 (1990).

[27] N. Kidonakis and J. Smith, hep-ph/9506253.

[28] W. Beenakker, H. Kuijf, W. L. van Neerven, and J. Smith, Phys. Rev. D 40, 54 (1989). 
[29] P. Nason, S. Dawson, and R. K. Ellis, Nucl. Phys. B303, 607 (1988).

[30] CTEQ Collaboration, H. L. Lai et al., Eur. Phys. J. C 12, 375 (2000).

[31] E. Laenen, J. Smith, and W. L. van Neerven, Phys. Lett. B 321, 254 (1994).

[32] H. Contopanagos and G. Sterman, Nucl. Phys. B419, 77 (1994).

[33] R. Hamberg, W. L. van Neerven, and T. Matsuura, Nucl. Phys. B359, 343 (1991).

[34] W. L. van Neerven and E. B. Zijlstra, Nucl. Phys. B382, 11 (1992).

[35] L. Magnea, Nucl. Phys. B349, 703 (1991).

[36] G. Sterman and W. Vogelsang, JHEP 0102, 016 (2001).

[37] N. Kidonakis and G. Sterman, in Results and Perspectives in Particle Physics, ed. M. Greco (INFN-Frascati), p. 333, hep-ph/9607222.

[38] M. Beneke and V. M. Braun, Nucl. Phys. B454, 253 (1995).

[39] G. Sterman and W. Vogelsang, in Proceedings of the International Europhysics Conference on High Energy Physics, eds. K. Huitu, H. Kurki-Suonio, J. Maalampi (IoP), p. 423, hepph/9910371.

[40] H. Lai and H. Li, Phys. Lett. B 471, 220 (1999).

[41] S. R. Blusk [CDF Collaboration], hep-ex/0009050.

[42] B. Abbott et al. [D0 Collaboration], Phys. Rev. Lett. 83, 1908 (1999).

[43] G. Oderda, N. Kidonakis, and G. Sterman, in EPIC 1999, p. 377, hep-ph/9906338.

[44] N. Kidonakis and J. Smith, Phys. Rev. D 51, 6092 (1995).

[45] N. Kidonakis, in DPF2000, hep-ph/0009013.

[46] N. Kidonakis and J. Smith, Mod. Phys. Lett. A 11, 587 (1996). 